Stochastic Systems

2013, Vol. 3, No. 2, 442-499

DOI: $10.1214 / 11-$ SSY041

\title{
BROWNIAN INVENTORY MODELS WITH CONVEX HOLDING COST, PART 1: AVERAGE-OPTIMAL CONTROLS*
}

\author{
By J. G. Dai and Dacheng Yao \\ Cornell University ${ }^{\dagger}$ and Chinese Academy of Sciences
}

\begin{abstract}
We consider an inventory system in which inventory level fluctuates as a Brownian motion in the absence of control. The inventory continuously accumulates cost at a rate that is a general convex function of the inventory level, which can be negative when there is a backlog. At any time, the inventory level can be adjusted by a positive or negative amount, which incurs a fixed cost and a proportional cost. The challenge is to find an adjustment policy that balances the holding cost and adjustment cost to minimize the long-run average cost. When both upward and downward fixed costs are positive, our problem is an impulse control problem. When both fixed costs are zero, our problem is a singular or instantaneous control problem. For the impulse control problem, we prove that a four-parameter control band policy is optimal among all feasible policies. For the singular control problem, we prove that a two-parameter control band policy is optimal.

We use a lower-bound approach, widely known as the "verification theorem", to prove the optimality of a control band policy for both the impulse and singular control problems. Our major contribution is to prove the existence of a "smooth" solution to the free boundary problem under some mild assumptions on the holding cost function. The existence proof leads naturally to a numerical algorithm to compute the optimal control band parameters. We demonstrate that the lower-bound approach also works for a Brownian inventory model which prohibits an inventory backlog. A companion paper ("Brownian inventory models with convex holding cost, part 2: discountoptimal controls", Stochastic Systems, Vol. 3, No. 2, pp. 500-573) explains how to adapt the lower-bound approach to study a Brownian inventory model under a discounted cost criterion.
\end{abstract}

Received October 2011.

* Research supported in part by NSF grants CMMI-0727400, CMMI-0825840, CMMI1030589, and CNS-1248117.

${ }^{\dagger}$ On leave from Georgia Institute of Technology.

AMS 2000 subject classifications: Primary 60J70, 90B05, 93E20.

Keywords and phrases: Impulse control, singular control, instantaneous control, control band, verification theorem, free boundary problem, smooth pasting, quasi-variational inequality. 
1. Introduction. This paper concerns the optimal control of Brownian inventory models under a long-run average cost criterion. It serves two purposes. First, it provides a tutorial on the powerful lower-bound approach, known as the "the verification theorem", for proving the optimality of a control band policy among all feasible policies. The tutorial is rigorous and self-contained with the exception of the standard Itô's formula. Second, it contributes to the literature by proving the existence of a "smooth" solution to the free boundary problem with a general convex holding cost function. The existence proof leads naturally to a numerical algorithm to compute the optimal control band parameters. The companion paper [33] studies the optimal control of Brownian inventory models under a discounted cost criterion.

The model description. In this paper and the companion paper [33], we assume that the inventory netflow process follows a Brownian motion with drift $\mu$ and variance $\sigma^{2}$. The netflow process captures the difference between regular supplies, possibly through a long term contract, and customer demands. Controls are exercised on the netflow process to maintain the inventory at desired positions. The controlled process, denoted by $Z=\{Z(t), t \geq 0\}$, is called the inventory process in this paper. For each time $t \geq 0, Z(t)$ is interpreted as the inventory level at time $t$, although $Z(t)$ can be negative, in which case $|Z(t)|$ represents the inventory backlog at time $t$. We assume that the holding cost function $h: \mathbb{R} \rightarrow \mathbb{R}_{+}$is a general convex function. Thus, $\int_{0}^{t} h(Z(s)) d s$ is the cumulative inventory cost by time $t$.

We also assume an adjustable, i.e. upward or downward, inventory position. All adjustments are realized immediately without any leadtime delay. Each upward adjustment with amount $\xi>0$ incurs a cost $K+k \xi$, where $K \geq 0$ and $k>0$ are the fixed cost and the variable cost rate, respectively, for each upward adjustment. Similarly, each downward adjustment with amount $\xi$ incurs a cost of $L+\ell \xi$ with fixed cost $L \geq 0$ and variable cost rate $\ell>0$. The objective is to find some control policy that balances the inventory cost and the adjustment cost so that the long-run average cost is minimized.

We use the inventory terminology from supply chain management to describe the Brownian control problems, although we also can describe them by using the terminology of cash flow management. In this case, $Z(t)$ represents the cash amount at time $t \geq 0$. A large number of papers in the economics literature have studied the Brownian control problems (e.g. Dixit [36]). Readers are referred to Stokey [74] and her references for economic applications of Brownian control problems. While the discounted cost criterion is appropri- 
ate for cash flow management, the long-run average cost criterion is natural for many production/inventory problems.

When both fixed costs $K$ and $L$ are positive, it is clear that non-trivial feasible control policies should limit the number of adjustments to be finite within any finite time interval. Under such a control policy, inventory is adjusted at a sequence of discrete times and the resulting control problem is termed the impulse control of a Brownian motion. When both fixed costs $K=0$ and $L=0$, it can be advantageous for the system to make an "infinitesimal" amount of adjustments at any moment. Indeed, as shown in Section 6, an optimal policy will make an uncountable number of adjustments within a finite time interval. The resulting control problem is termed the singular or instantaneous control of a Brownian motion. In this paper, we treat impulse and singular control of a Brownian motion in a single framework. Conceptually, we can view the singular control problem as a limit of a sequence of impulse control problems as fixed costs $K \downarrow 0$ and $L \downarrow 0$. Such a connection between impulse and singular control problems allows us to solve a mixed impulse-singular control problem (for example, $K>0$ and $L=0$ ) with less effort.

Non-linear holding cost. When holding cost function $h$ is given by

$$
h(x)= \begin{cases}-p x & \text { if } x<0 \\ c x & \text { if } x \geq 0\end{cases}
$$

for some constants $p>0$ and $c>0$, we call $h$ in (1.1) a linear holding cost function, even though $h(x)$ in (1.1) is piecewise linear in inventory level $x$. With this holding cost function, both the inventory backlog cost and the inventory excess cost are linear, but $h(x)$ is not differentiable at $x=0$. Although many papers focus on the linear holding cost function (e.g. [43]), there are many applications that motivate the non-linear holding cost function. For example, [24] and [69] studied optimal index tracking of a benchmark index when there are transaction costs. An impulse control problem with quadratic holding cost arises naturally in their studies. Quadratic holding cost and general convex holding cost also arise, for example, in $[22,68,78]$.

Optimal policy structure. For an impulse Brownian control problem under the long-run average cost criterion, we prove in Section 5 that control band policy $\varphi=\{d, D, U, u\}$ with $d \leq D \leq U \leq u$ is optimal among all feasible policies. Under the control band policy $\varphi$, an adjustment is placed to bring the inventory up to level $D$ when the inventory level drops to level $d$ 
and to bring the inventory down to level $U$ when the inventory level rises to level $u$. For a singular Brownian control problem, we show in Section 6 that the optimal policy is a degenerate control band policy with two free parameters $D=d$ and $U=u$. When the inventory level is restricted to be always nonnegative, we show in Section 7 that the optimal policy for an impulse Brownian control problem is again a control band policy. Depending on holding cost function $h$, this control band policy sometimes, but not always, has only three free parameters $D, U$ and $u$ that need to be characterized with the lowest boundary $d=0$. Although this paper does not explicitly study mixed impulse-singular Brownian control problems, it is clear from our proofs that a degenerate control band policy with three parameters is optimal.

The lower-bound approach and the free boundary problem. This paper promotes a three-step, lower bound approach to solving Brownian control problems under the long-run average cost criterion. In the first step, we prove Theorem 4.1 to show that if there exist a constant $\gamma$ and a "smooth" test function $f$ that is defined on the entire real line (or the positive half line when inventory is not allowed to be backlogged) such that $f$ and $\gamma$ jointly satisfy some differential inequalities, then the long-run average cost under any feasible policy is at least $\gamma$. We formulate and prove this theorem for all impulse, singular and mixed impulse-singular control problems. In the second step, we show in Theorems 5.1 and 6.1 that for a given control band policy, its long-run average cost can be computed as a solution to a Poisson equation. This equation is a second order ordinary differential equation (ODE) with given boundary conditions at the boundary points of the band. As a part of the solution to the Poisson equation, we also obtain the relative value function. The relative value function can be extended naturally to the entire real line, but the extended function may not be continuously differentiable at the boundary points of the control band. In the third step, we search for a control band policy such that the corresponding relative value function can indeed be extended smoothly as a function $f$ on the entire real line. Furthermore, this smooth function $f$, together with the long-run average cost under the control band policy, satisfies the differential inequalities in step 1 within the entire real line. Clearly, if the control band policy in step 3 can be found, it must be an optimal policy by Theorem 4.1. The lower-bound theorem, Theorem 4.1, is known as the "verification theorem" in the literature.

Step 3 is the most critical step in our three-step approach. In order to make the relative value function smoothly extendible to the entire real line, the parameters of the control band, which serve as the boundary points of 
the ODE, must be carefully selected. The smoothness requirements impose conditions of the ODE solution at these yet to be determined boundary points. Thus, we call the ODE in step 3 the free boundary ODE problem. Solving the free boundary problem to find the optimal parameters is also known as the "smooth pasting" method [22]. Solving a free boundary problem can be difficult technically, since the number of free parameters of an optimal control band policy dictates the level of difficulty in solving the problem. Many papers have left it unsolved (e.g. [36, 71]), assuming there is a solution to the free boundary problem with a certain smoothness property.

Contributions. The Brownian inventory control problem is now a classical problem, starting with Bather [13] in 1966. We briefly survey the research area below. In addition to providing a self-contained tutorial on the lowerbound approach to studying optimal control problems, our paper contributes significantly in the following areas. (a) Under a general convex holding cost function with some minor assumptions, we rigorously prove the existence of a control band policy that is optimal for both the impulse and singular control problems under the long-run average cost criterion. (b) Under the general convex holding cost function, we prove the existence of a solution to the four-parameter free-boundary problem. Our existence proof leads naturally to algorithms for computing optimal control band parameters, which reduce to root findings for continuous, monotone functions. Thus, the convergence of these algorithms are guaranteed. A survey of the literature did not reveal the existence of a solution to the four-parameter free boundary problem under the long-run average cost criterion. In the discounted setting, [31] solved the four-parameter free boundary problem when $h$ is linear, and [10] solved the problem when $h$ is quadratic. Recently, Feng and Muthuraman [39] developed an algorithm to numerically solve the four-parameter free boundary problem for the discounted Brownian control problem. They illustrated the convergence of their algorithm through some numerical examples. (c) Under the long-run average cost criterion, our lower-bound approach provides a unified treatment for both the impulse and singular control problems, with and without inventory backlog. In particular, we do not need to employ the vanishing discount approach [38, 46, 73] to study the long-run average cost problems. In [74], Stokey summarized both the impulse and instantaneous controls of Brownian motion with a general convex holding cost function, focusing on the discounted cost problems and employing the vanishing discount approach to deal with the long-run average cost problems. It is appealing that our current paper studies the long-run average cost problem directly, and characterizes the optimal parameters directly without going through the vanishing discount procedure. 
Literature review. We first give a sample of major application areas of singular and impulse Browian controls. We then survey the main methodologies for studying these control problems including the latest research development when the driving process is a diffusion process or Levy process. As stated, one purpose of this paper and the companion paper [33] is to serve as a tutorial; we do not attempt to conduct a comprehensive survey. Unless stated otherwise, we restrict our discussions to one-dimensional control problems.

Singular Brownian control has been used to study storage systems [44, 45, 76], multiclass make-to-stock production systems [79, 81] with additional features such as subcontracting [23] or by-products and random yield [66] or parallel server systems with outsourcing and order cancellation, maketo-order production systems [7, 9], queues with input control and customer abandonment [72, 80], product launching and advertising [57], and stochastic processing networks under a complete resource pooling condition $[8,15,16$, $32,41,42,59]$. Singular control with discretionary stopping has been studied in $[35,61]$. In addition to the economic papers cited in this introduction, impulse Brownian control has been used to study manufacturing systems [53], paper plants for paper making and cutting [54], natural resource economics such as forest harvesting problems $[2,3,4,5]$, inventory control problems $[12,18,21,28,65,75]$, and financial problems such as dividend problems [6, 27, 49, 67], inflation and pricing problems [78], portfolio optimization problems with transaction costs $[24,47,51,63,77]$, and exchange rate problems $[26,48,62]$. See [52] for a survey on using impulse control to solve these financial problems.

The lower-bound approach has been used in [65, 76] under a long-run average cost criterion and in $[43,44]$ under a discounted cost criterion. The approach is essentially the same as the quasi-variational inequality (QVI) approach pioneered by Bensoussan and Lions [19]. The QVI approach was systematically developed in a French book, which was later translated into English (see [20]). An appealing feature of the QVI approach is that it is sufficient to solve a QVI problem in order to obtain an optimal policy for an inventory control problem, and this sufficiency is established in [20]. The QVI problem is a pure analytical problem that relates closely to the free boundary problem. Many authors start with the QVI problems, relying on the verification theorem developed in [20]; see for example, [17, 18, 21, 75]. The potential drawback of this approach is that a slight difference in the formulation of a Brownian or diffusion control problem from the setting in [20] may require developing a new verification theorem, presumably mimicking the development in reference. In contrast, our lower-bound approach 
provides a self-contained, rigorous proof simultaneously for impulse, singular and mixed control problems. It also allows us to observe first-hand how the smoothness requirement of a solution to the free-boundary problem is used. We also believe the lower-bound approach is easier to generalize to high dimensional Brownian control problems.

The impulse control problem with both upward and downward adjustments was studied as early as 1976 and 1978 by Constantinides [30] and Constantinides et al. [31]. The first paper studied the long-run average cost objective and the second paper studied the discounted cost objective. Both papers assume the holding cost function is linear as given in (1.1). Under this holding cost function, the optimal control band parameters can be explicitly characterized. Baccarin [10] studied the discounted impulse Brownian control problem with a quadratic inventory cost function. When the inventory is restricted to be nonnegative, but still under the linear holding cost assumption (1.1), Harrison et al. [43] studied the discounted cost, impulse Brownian control problem whereas Ormeci et al. [65] studied the long-run average cost problem. Under the linear holding cost function assumption, the optimal policy is a degenerate control band policy $\{0, D, U, u\}$, where three optimal parameters $D, U, u$ can be determined explicitly. However, under our general convex holding cost assumption, the optimal policy for the impulse control problem without inventory backlog is again a control band policy $\{d, D, U, u\}$, with $d$ sometimes being strictly positive. Harrison and Taksar [44] and Taksar [76] studied the singular Brownian control problem under a general convex inventory cost function assumption. The former paper studied the discounted cost problem and the latter studied the long-run average cost problem. Taksar [76] characterized the optimal control band parameters through the optimal stopping time to a stochastic game without solving the two-parameter free boundary problem. As in [76], Stokey [74] characterized the optimal parameters through a stopping time problem without solving the four-parameter free boundary problem. These stopping time characterizations do not easily lead to any numerical algorithm to compute optimal parameters. Kumar and Muthuraman [55] developed a numerical algorithm to solve high-dimensional singular control problems.

In his pioneering paper, Bather [13] studied the impulse Brownian motion control problem without downward adjustment under the long-run average cost criterion. For most inventory problems, without downward adjustment is a natural setting. Under a general holding cost function, the author suggested that an $(s, S)$ policy is optimal and derived equations that characterize the optimal parameters $s$ and $S$. Many authors have generalized Bather's work to discounted cost problems with linear holding cost in [75], 
to discounted cost problems with and without inventory backlog in [28], to discounted cost problems under the general convex holding cost function assumption in [17], to discounted cost problems with positive constant leadtime in [12], and to compound Poisson and diffusion demand processes in $[18,21]$. Because there is no downward adjustment in these problems, the optimal policy has two parameters and the resulting two-parameter free boundary problem can be solved more easily than the four-parameter one.

As mentioned, most literature assumes that the driving process (the uncontrolled state process) is a Brownian motion. Therefore, this paper and the companion paper [33] adopt the same framework to keep the tutorial accessible. All of these papers prove that a control band policy is optimal. Motivated by various applications, other driving processes have been used to study these control problems. A control band policy continues to be optimal when the driving process is a geometric Brownian motion [29, 47, 58, 60, 63] or a mean-reverting diffusion process $[25,27]$. In $[1,14,57]$, the authors studied singular control problems when the driving process is a general diffusion process, and in $[6,37,50,67]$, the authors studied impulse control problems when the driving processes are general diffusion processes. The latter group of papers assume that the state is restricted to be nonnegative and only downward adjustment is allowed. For example, under their Assumption 2.1, Alvarez and Lempa [6] proved the existence of a unique pair of parameters $U^{*}<u^{*}$ such that the control band policy associated with the pair is optimal among all feasible policies. Their approach is closely related to, but different from, the smooth-pasting one outlined earlier in this introduction, i.e., (a) for each pair of parameters $(U, u)$, derive an expression for the value function of the two-parameter control band policy associated with $(U, u)$; (b) solve a non-linear optimization problem in two variables $U$ and $u$; prove that under Assumption 2.1 there is a unique pair $\left(U^{*}, u^{*}\right)$ that optimizes the value function; and (c) prove that the value function associated with $\left(U^{*}, u^{*}\right)$ satisfies all the conditions of a verification theorem. Their Assumption 2.1 proves to be strong; it rules out, for example, the quadratic holding cost function when the driving process is a Brownian motion, a geometric Brownian motion, or a mean-riveting process. Egami [37] adopted another approach. It is known that an impulse control problem is equivalent to a sequence of optimal stopping problems; see, for example, Davis [34] and Oksendal and Sulem [64]. Each optimal stopping problem is of the form

$$
\Pi(x)=\sup _{\tau \geq 0} \mathbb{E}_{x}\left[e^{-\alpha \tau} \pi\left(X_{\tau}\right)\right],
$$

where $\tau$ is a stopping time, $\alpha \geq 0$ is a discount factor and $\pi$ is a terminal cost function. Such an optimal problem has been solved by Dayanika and 
Karatzas (2003). In particular, the optimal stopping time is given by

$$
\tau^{*}=\inf \left\{t \geq 0: X_{t} \in S\right\}, \text { where } S=\{x: \Pi(x)=\pi(x)\} .
$$

Egami [37] proved that the value function $V(x)$ of the (discounted) impulse control problem is related to the value function of an optimal stopping problem in (1.2) whose terminal cost $\pi$ is equal to a function of the unknown value function $V(x)$. Under a certain assumption on problem data, the author was able to prove beautifully that the corresponding set $S$ in (1.3) is a singleton, without explicitly solving the unknown value function $V$. Thus, the structure of an optimal policy is again a control band policy, without invoking a verification theorem. Egami [37] claimed without proof that an impulse problem with both upward and downward adjustment can also be solved. (It is interesting to see if this impulse control problem can indeed be solved as the four-parameter non-linear optimization problem will be significantly more difficult to solve.) It remains to be seen whether [37] can be extended to average cost problems. In [11] and [56], the authors studied impulse control problems when the driving process is a Levy process. Lakner and Reed [56] proved a verification theorem, but did not solve the free boundary problems. Benkherouf and Bensoussan [18] and Bensoussan et al. [21] studied the problem without downward adjustment when the Levy process is a sum of a Brownian motion and a compound Poisson process. In [21], a control band policy $(d, D)$ was proven optimal when the jump size is exponentially distributed and in [18] when the jump size has a general distribution.

Paper organization. The remainder of this paper is organized as follows. In Section 2, we define our Brownian control problem in a unified setting that includes impulse, singular and mixed impulse-singular controls. In Section 3, we present a version of the Itô's formula that does not require the test function $f$ be $C^{2}$ function. A lower bound for all feasible policies is established in Section 4. Section 5 discusses impulse control problems that allow inventory backlog under the long-run average cost criterion. Section 5.1 shows that under a control band policy, a Poisson equation can produce a solution that gives both the long-run average cost and the corresponding relative value function. Under the assumption that a free-boundary problem has a unique solution that has desired regularity properties, Section 5.2 proves that there is a control band policy whose long-run average cost achieves the lower bound. Thus, the control band policy is optimal among all feasible policies. Section 5.3 details the proof of the solution to the freeboundary problem and characterizes the parameters for the optimal control 
band policy. Section 5.3 constitutes the main technical contribution of this paper. Section 6 , essentially a special case of Section 5 when both $K=0$ and $L=0$, solves the singular control problem. Section 7 describes impulse control problems when inventory backlog is prohibited. Section 8 summarizes this paper and suggests an extension.

2. Brownian control models. Let $X=\{X(t), t \geq 0\}$ be a Brownian motion with drift $\mu$ and variance $\sigma^{2}$, starting from $x$. Then, $X$ has the following representation

$$
X(t)=x+\mu t+\sigma W(t), \quad t \geq 0,
$$

where $W=\{W(t), t \geq 0\}$ is a standard Brownian motion that has drift 0 , variance 1 , starting from 0 . We assume $W$ is defined on some filtered probability space $\left(\Omega,\left\{\mathcal{F}_{t}\right\}, \mathcal{F}, \mathbb{P}\right)$ and $W$ is an $\left\{\mathcal{F}_{t}\right\}$-martingale. Thus, $W$ is also known as an $\left\{\mathcal{F}_{t}\right\}$-standard Brownian motion. We use $X$ to model the netflow process of an inventory system. For each $t \geq 0, X(t)$ represents the inventory level at time $t$ if no control has been exercised by time $t$. The netflow process will be controlled and the actual inventory level at time $t$, after controls have been exercised, is denoted by $Z(t)$. The controlled process is denoted by $Z=\{Z(t), t \geq 0\}$. With a slight abuse of terminology, we call $Z(t)$ the inventory level at time $t$, although when $Z(t)<0,|Z(t)|$ is the backorder level at time $t$.

Controls are dictated by a policy $\varphi$, which is a pair of stochastic processes $\left(Y_{1}, Y_{2}\right)$ satisfying the following three properties: (a) for each sample path $\omega \in \Omega, Y_{i}(\omega, \cdot) \in \mathbb{D}$, where $\mathbb{D}$ is the set of functions on $\mathbb{R}_{+}=[0, \infty)$ that are right continuous on $[0, \infty)$ and have left limits in $(0, \infty)$; (b) for each $\omega$, $Y_{i}(\omega, \cdot)$ is a nondecreasing function; and (c) $Y_{i}$ is adapted to the filtration $\left\{\mathcal{F}_{t}\right\}$, namely, $Y_{i}(t)$ is $\mathcal{F}_{t}$-measurable for each $t \geq 0, i=1,2$. We call $Y_{1}(t)$ and $Y_{2}(t)$ the cumulative upward and downward adjustment, respectively, of the inventory in $[0, t]$. Under policy $\left(Y_{1}, Y_{2}\right)$, the inventory level at time $t$ is given by

$$
Z(t)=X(t)+Y_{1}(t)-Y_{2}(t)=x+\sigma W(t)+\mu t+Y_{1}(t)-Y_{2}(t), \quad t \geq 0 .
$$

Therefore, $Z$ is a semimartingale, namely, a martingale $\sigma W$ plus a process that is of bounded variation.

A point $t \geq 0$ is said to be an increasing point of $Y_{1}$ if $Y_{1}(s)-Y_{1}(t-)>0$ for each $s>t$, where $Y_{1}(t-)$ is the left limit of $Y_{1}$ at $t$ with convention that $Y_{1}(0-)=0$. When $t$ is an increasing point of $Y_{1}$, we call it an upward adjustment time. Similarly, we define an increasing point of $Y_{2}$ and call it a 
downward adjustment time. Let $N_{i}(t)$ be the cardinality of the set

$$
\left\{s \in[0, t]: Y_{i} \text { increases at } s\right\}, \quad i=1,2 .
$$

In general, we allow an upward or downward adjustment at time $t=0$. By convention, we set $Z\left(0_{-}\right)=x$ and call $Z\left(0_{-}\right)$the initial inventory level. By $(2.1)$,

$$
Z(0)=x+Y_{1}(0)-Y_{2}(0)
$$

which can be different from the initial inventory level $Z(0-)$.

The two types of costs associated with a control are fixed costs and proportional costs. We assume that each upward adjustment incurs a fixed cost of $K \geq 0$ and each downward adjustment incurs a fixed cost of $L \geq 0$. In addition, each unit of upward adjustment incurs a proportional cost of $k>0$ and each unit of downward adjustment incurs a proportional cost of $\ell>0$. Thus, by time $t$, the system incurs the cumulative proportional cost $k Y_{1}(t)$ for upward adjustment and the cumulative proportional cost $\ell Y_{2}(t)$ for downward adjustment. When $K>0$, we are only interested in policies such that $N_{1}(t)<\infty$ for each $t>0$; otherwise, the total cost would be infinite in the time interval $[0, t]$. Thus, when $K>0$, we restrict upward controls that have a finitely many upward adjustment in a finite interval. This is equivalent to requiring $Y_{1}$ to be a piecewise constant function on each sample path. Under such an upward control, we can list the upward adjustment times as a discrete sequence $\left\{T_{1}(n): n \geq 0\right\}$, where the $n$th upward adjustment time can be defined recursively via

$$
T_{1}(n)=\inf \left\{t>T_{1}(n-1): \Delta Y_{1}(t)>0\right\},
$$

where, by convention, $T_{1}(0)=0$ and $\Delta Y_{1}(t)=Y_{1}(t)-Y_{1}(t-)$. The amount of the $n$th upward adjustment is denoted by

$$
\xi_{1}(n)=Y_{1}\left(T_{1}(n)\right)-Y_{1}\left(T_{1}(n)-\right) \quad n=0,1, \ldots
$$

It is clear that specifying such an upward adjustment policy $Y_{1}=\left\{Y_{1}(t), t \geq\right.$ $0\}$ is equivalent to specifying a sequence of $\left\{\left(T_{1}(n), \xi_{1}(n)\right): n \geq 0\right\}$. In particular, given the sequence, we have

$$
Y_{1}(t)=\sum_{i=0}^{N_{1}(t)} \xi_{1}(i),
$$

and $N_{1}(t)=\max \left\{n \geq 0: T_{1}(n) \leq t\right\}$. Thus, when $K>0$, it is sufficient to specify the sequence $\left\{\left(T_{1}(n), \xi_{1}(n)\right): n \geq 0\right\}$ to describe an upward 
adjustment policy. Similarly, when $L>0$, it is sufficient to specify the sequence $\left\{\left(T_{2}(n), \xi_{2}(n)\right): n \geq 0\right\}$ to describe a downward adjustment policy and

$$
Y_{2}(t)=-\sum_{i=0}^{N_{2}(t)} \xi_{2}(i) .
$$

Merging these two sequences gives the sequence $\left\{\left(T_{n}, \xi_{n}\right), n \geq 0\right\}$, where $T_{n}$ is the $n$th adjustment time of the inventory and $\xi_{n}$ is the amount of adjustment at time $T_{n}$. When $\xi_{n}>0$, the $n$th adjustment is an upward adjustment and when $\xi_{n}<0$, the $n$th adjustment is a downward adjustment. The policy $\left(Y_{1}, Y_{2}\right)$ is adapted if $T_{n}$ is an $\left\{\mathcal{F}_{t}\right\}$-stopping time and each adjustment $\xi_{n}$ is $\mathscr{F}_{T_{n}-}$ measurable.

In addition to the adjustment cost, we assume the system incurs a holding cost at rate $h(x)$ : when the inventory level is at $Z(t)=x$, the system incurs a cost of $h(x)$ per unit of time. Therefore, the cumulative holding cost in $[0, t]$ is

$$
\int_{0}^{t} h(Z(s)) d s
$$

Under a feasible policy $\varphi=\left\{\left(Y_{1}(t), Y_{2}(t)\right)\right\}$ with initial inventory level $Z(0-)=x$, the long-run average cost $\mathrm{AC}(x, \varphi)$ is

$$
\limsup _{t \rightarrow \infty} \frac{1}{t} \mathbb{E}_{x}\left[\int_{0}^{t} h(Z(s)) d s+K N_{1}(t)+L N_{2}(t)+k Y_{1}(t)+\ell Y_{2}(t)\right],
$$

where $\mathbb{E}_{x}$ is the expectation operator conditioning the initial inventory level $Z(0-)=x$. As mentioned, when $K>0$ and $L>0$, it is sufficient to restrict feasible policies to be the impulse type given in (2.2) and (2.3). A Brownian control model with controls limited to impulse type is called the impulse Brownian control model. When $K=0$ and $L=0$, under an optimal policy, $N_{1}(t)=\infty$ and $N_{2}(t)=\infty$ with positive probability for each $t>0$. We call the corresponding control problem the instantaneous Brownian control model or singular Brownian control model.

For holding cost $h: \mathbb{R} \rightarrow \mathbb{R}_{+}$, we make the following assumption.

Assumption 1. Assume that the continuous holding cost function $h$ : $\mathbb{R} \rightarrow \mathbb{R}^{+}$satisfies the following conditions: (a) it is convex; (b) there exists an $a$ such that $h \in C^{2}(\mathbb{R})$ except at $a$, and $h(a)=0 ;\left(\right.$ c) $h^{\prime}(x)<0$ for $x<a$ and $h^{\prime}(x)>0$ for $x>a$; and (d) When $\lambda=\frac{2 \mu}{\sigma^{2}} \neq 0, h^{\prime}(x)$ has a smaller order than $e^{-\lambda x}$, that is

$$
\int_{-\infty}^{a}\left|h^{\prime}(y)\right| e^{\lambda(y-a)} d y<\infty \text { if } \lambda=\frac{2 \mu}{\sigma^{2}}>0
$$


and

$$
\int_{a}^{\infty}\left|h^{\prime}(y)\right| e^{\lambda(y-a)} d y<\infty \text { if } \lambda=\frac{2 \mu}{\sigma^{2}}<0 .
$$

Remark. (i) $h(x)$ in (1.1) and $h(x)=x^{2}$ all satisfy Assumption 1. (ii) Under Assumption 1 (c), the convexity of $h$ implies that $\lim _{|x| \rightarrow \infty} h(x)=\infty$.

We only consider feasible policies that satisfy

$$
\begin{aligned}
& \mathbb{E}_{x}\left[Y_{i}(t)\right]<\infty \quad i=1,2, \\
& \mathbb{E}_{x}\left[N_{1}(t)\right]<\infty \text { when } K>0 \text { and } \quad \mathbb{E}_{x}\left[N_{2}(t)\right]<\infty \text { when } L>0
\end{aligned}
$$

for each $t \geq 0$. Otherwise, $\operatorname{AC}(x, \varphi)=\infty$. Note that some applications may always require a nonnegative inventory level, namely,

$$
Z(t) \geq 0 \text { for } t \geq 0
$$

3. The Itô's formula. In this section, we first state a version of Itô's formula. We then provide a lower bound result for the long-run average cost in (2.4). Recall that for a function $g \in \mathbb{D}$, it is right continuous on $[0, \infty)$ and has left limits in $(0, \infty)$. We use $g^{c}$ to denote the continuous part of $g$, namely,

$$
g^{c}(t)=g(t)-\sum_{0 \leq s \leq t} \Delta g(s) \quad \text { for } t \geq 0 .
$$

Here, we assume $g(0-)$ is well defined. Recall that under any feasible policy $\varphi=\left(Y_{1}, Y_{2}\right)$, the inventory process $Z=\{Z(t): t \geq 0\}$ has the semimartingale representation (2.1). Because Brownian motion has continuous sample paths, we have

$$
Z^{c}(t)=X(t)+Y_{1}^{c}(t)-Y_{2}^{c}(t) \quad \text { for } t \geq 0
$$

Lemma 3.1. Assume that $f \in C^{1}(\mathbb{R})$ and $f^{\prime}$ is absolutely continuous such that $f^{\prime}(b)-f^{\prime}(a)=\int_{a}^{b} f^{\prime \prime}(u) d u$ for any $a<b$ with $f^{\prime \prime}$ locally in $L^{1}$. Then

$$
\begin{aligned}
f(Z(t))= & f(Z(0))+\int_{0}^{t} \Gamma f(Z(s)) d s \\
& +\sigma \int_{0}^{t} f^{\prime}(Z(s)) d W(s)+\int_{0}^{t} f^{\prime}(Z(s-)) d Y_{1}^{c}(s) \\
& -\int_{0}^{t} f^{\prime}(Z(s-)) d Y_{2}^{c}(s)+\sum_{0<s \leq t} \Delta f(Z(s))
\end{aligned}
$$


where

$$
\Gamma f(x)=\frac{1}{2} \sigma^{2} f^{\prime \prime}(x)+\mu f^{\prime}(x) \text { for each } x \in \mathbb{R} \text { such that } f^{\prime \prime}(x) \text { exists, }
$$

is the generator of the $\left(\mu, \sigma^{2}\right)$-Brownian motion $X$ and $\int_{0}^{t} f^{\prime}(Z(s)) d W(s)$ is interpreted as the Itô integral.

Remark. Although $f^{\prime \prime}(u)$ is only defined on almost all $u$ in $\mathbb{R}, \int_{0}^{t} f^{\prime \prime}(Z(s)) d s$ is uniquely defined almost surely. Indeed,

$$
\sigma^{2} \int_{0}^{t} f^{\prime \prime}(Z(s)) d s=\frac{1}{2} \int_{\mathbb{R}} f^{\prime \prime}(a) L^{a}(t) d a,
$$

where $L^{a}$ is the local time of $Z$ at $a$.

Proof. For any semimartingale $Z$, from Theorem 71 of [70, pp. 221] and the comment of [70, pp. 70], it follows that

$$
\begin{aligned}
f(Z(t))= & f(Z(0))+\int_{0}^{t} f^{\prime}(Z(s-)) d Z^{c}(s) \\
& +\frac{1}{2} \int_{0}^{t} f^{\prime \prime}(Z(s-)) d\left[Z^{c}, Z^{c}\right](s)+\sum_{0<s \leq t} \Delta f(Z(s)),
\end{aligned}
$$

where $\left[Z^{c}, Z^{c}\right]$ is the quadratic variation of $Z^{c}$. Using semimartingale representation (3.1), we have

$$
\left[Z^{c}, Z^{c}\right](t)=[X, X](t)=\sigma^{2} t
$$

and

$$
\begin{gathered}
\int_{0}^{t} f^{\prime}(Z(s-)) d Z^{c}(s)=\int_{0}^{t} f^{\prime}(Z(s-)) \mu d s+\int_{0}^{t} f^{\prime}(Z(s-)) \sigma d W(s) \\
+\int_{0}^{t} f^{\prime}(Z(s-)) d Y_{1}^{c}(s)-\int_{0}^{t} f^{\prime}(Z(s-)) d Y_{2}^{c}(s)
\end{gathered}
$$

for $t \geq 0$. Because $Y_{1}$ and $Y_{2}$ have at most countably many jump points, $Z$ has at most countably many discontinuity points. Therefore,

$$
\begin{aligned}
\int_{0}^{t} f^{\prime}(Z(s-)) d s & =\int_{0}^{t} f^{\prime}(Z(s)) d s \text { and } \\
\int_{0}^{t} f^{\prime}(Z(s-)) d W(s) & =\int_{0}^{t} f^{\prime}(Z(s)) d W(s)
\end{aligned}
$$

for all $t \geq 0$ almost surely. Itô's formula (3.2) then follows from (3.4)-(3.8). 
4. Lower bound. Next, we state and prove a theorem that establishes a lower bound for the optimal long-run average cost. This theorem is closely related to the verification theorem in the literature. Its proof is self-contained, using the Itô lemma in Section 3.

THEOREM 4.1. Suppose that $f \in C^{1}(\mathbb{R})$ and $f^{\prime}$ is absolutely continuous such that $f^{\prime \prime}$ is locally $L^{1}$. Suppose there exists a constant $M>0$ such that $\left|f^{\prime}(x)\right| \leq M$ for all $x \in \mathbb{R}$. Assume further

$$
\begin{aligned}
& \Gamma f(x)+h(x) \geq \gamma \quad \text { for almost all } x \in \mathbb{R}, \\
& f(y)-f(x) \leq K+k(x-y) \quad \text { for } y<x, \\
& f(y)-f(x) \leq L+\ell(y-x) \quad \text { for } x<y,
\end{aligned}
$$

where $\Gamma$ is the generator defined in (3.3). Then $A C(x, \varphi) \geq \gamma$ for each feasible policy $\varphi$ and each initial state $x \in \mathbb{R}$.

Remark. (i) When $K=0$, condition (4.2) is equivalent to $f^{\prime}(x) \geq-k$ for each $x \in \mathbb{R}$. When $L=0$, condition (4.3) is equivalent to $f^{\prime}(x) \leq \ell$ for each $x \in \mathbb{R}$. (ii) Under an arbitrary control policy, the inventory level $Z$ can potentially reach any level. Thus, we require function $f$ to be defined on the entire real line $\mathbb{R}$. It is not enough to have $f$ defined on a certain interval $[d, u]$.

Proof. Let $\varphi=\left(Y_{1}, Y_{2}\right)$ be a feasible policy. We choose a version of $f^{\prime \prime}(x)$ such that (4.1) holds for every $x \in \mathbb{R}$. By Itô's formula (3.2),

$$
\begin{aligned}
f(Z(t))= & f(Z(0-))+\int_{0}^{t} \Gamma f(Z(s)) d s+\sigma \int_{0}^{t} f^{\prime}(Z(s)) d W(s) \\
& +\int_{0}^{t} f^{\prime}(Z(s-)) d Y_{1}^{c}(s)-\int_{0}^{t} f^{\prime}(Z(s-)) d Y_{2}^{c}(s) \\
& +\sum_{0 \leq s \leq t} \Delta f(Z(s)) \\
\geq & f(Z(0-))+\gamma t-\int_{0}^{t} h(Z(s)) d s+\sigma \int_{0}^{t} f^{\prime}(Z(s)) d W(s) \\
& +\int_{0}^{t} f^{\prime}(Z(s-)) d Y_{1}^{c}(s)-\int_{0}^{t} f^{\prime}(Z(s-)) d Y_{2}^{c}(s) \\
& +\sum_{0 \leq s \leq t} \Delta f(Z(s))
\end{aligned}
$$


where the inequality is due to (4.1). In the remainder of the proof, we separate into different cases depending on the positivity of $K$ and $L$. We also provide a complete proof for the case when $K>0$ and $L>0$ as well as sketches for proofs in other cases.

Case I: Assume that $K>0$ and $L>0$. In this case, it is sufficient to restrict feasible policies to impulse control policies $\left\{\left(T_{n}, \xi_{n}\right): n=0,1, \ldots\right\}$. In this case, $Y_{1}^{c}=0$ and $Y_{2}^{c}=0$. Conditions (4.2) and (4.3) imply that $\Delta f(Z(T(n))) \geq-\phi\left(\xi_{n}\right)$ for $n=0,1, \ldots$, where

$$
\phi(\xi)= \begin{cases}K+k \xi & \text { if } \xi>0 \\ 0 & \text { if } \xi=0 \\ L-l \xi & \text { if } \xi<0\end{cases}
$$

Therefore, (4.4) leads to

$$
\begin{gathered}
f(Z(t)) \geq f(Z(0-))+\gamma t-\int_{0}^{t} h(Z(s)) d s \\
+\sigma \int_{0}^{t} f^{\prime}(Z(s)) d W(s)-\sum_{n=0}^{N(t)} \phi\left(\xi_{n}\right)
\end{gathered}
$$

for each $t \geq 0$. Fix an $x \in \mathbb{R}$. We assume that

$$
\mathbb{E}_{x}\left(\int_{0}^{t} h(Z(s)) d s+\sum_{n=0}^{N(t)} \phi\left(\xi_{n}\right)\right)<\infty
$$

for each $t>0$. Otherwise, $\operatorname{AC}(x, \varphi)=\infty$ and thus $\operatorname{AC}(x, \varphi) \geq \gamma$ is trivially satisfied. Because $\left|f^{\prime}(x)\right| \leq M, \mathbb{E}_{x}\left|\int_{0}^{t} f^{\prime}(Z(s)) d W(t)\right|<\infty$ and $\mathbb{E}_{x} \int_{0}^{t} f^{\prime}(Z(s)) d W(s)=0$. Meanwhile

$$
f(Z(t)) \leq(f(Z(t)))^{+}
$$

and $\mathbb{E}_{x}\left[(f(Z(t)))^{+}\right]$is well defined, although it can be $\infty$, where, for a $b \in \mathbb{R}$, $b^{+}=\max (b, 0)$. Taking $\mathbb{E}_{x}$ on both sides of (4.6), we have

$$
\mathbb{E}_{x}\left[(f(Z(t)))^{+}\right] \geq \mathbb{E}_{x}[f(Z(0-))]+\gamma t-\mathbb{E}_{x}\left(\int_{0}^{t} h(Z(s)) d s+\sum_{n=0}^{N(t)} \phi\left(\xi_{n}\right)\right) .
$$

Dividing both sides by $t$ and taking the limit as $t \rightarrow \infty$, we have

$$
\liminf _{t \rightarrow \infty} \frac{1}{t}\left[\mathbb{E}_{x}\left(\int_{0}^{t} h(Z(s)) d s+\sum_{n=0}^{N(t)} \phi\left(\xi_{n}\right)\right)+\mathbb{E}_{x}\left[(f(Z(t)))^{+}\right]\right] \geq \gamma
$$


We consider two cases. In the first case when

$$
\liminf _{t \rightarrow \infty} \frac{1}{t} \mathbb{E}_{x}\left[(f(Z(t)))^{+}\right]=0
$$

it is clear that (4.7) implies the theorem. Now we consider the case when

$$
\liminf _{t \rightarrow \infty} \frac{1}{t} \mathbb{E}_{x}\left[(f(Z(t)))^{+}\right]=b>0 .
$$

It follows that for sufficiently large $t$,

$$
\mathbb{E}_{x}\left[(f(Z(t)))^{+}\right] \geq(b / 2) t
$$

Because $\left|f^{\prime}(y)\right| \leq M$, for all $y \in \mathbb{R}$,

$$
\left(f\left(y_{1}\right)\right)^{+}-\left(f\left(y_{2}\right)\right)^{+} \leq\left|f\left(y_{1}\right)-f\left(y_{2}\right)\right| \leq M\left|y_{1}-y_{2}\right| \leq M\left(\left|y_{1}\right|+\left|y_{2}\right|\right) .
$$

Therefore,

$$
|Z(t)| \geq \frac{1}{M}\left(f(Z(t))^{+}-(f(Z(0)))^{+}\right)-|Z(0)|,
$$

which, together with (4.8), implies

$$
\begin{aligned}
\mathbb{E}_{x}|Z(t)| & \geq \frac{1}{M}\left(\mathbb{E}_{x}\left[(f(Z(t)))^{+}\right]-\mathbb{E}_{x}\left[(f(Z(0)))^{+}\right]\right)-\mathbb{E}_{x}|Z(0)| \\
& \geq \frac{1}{M}\left((b / 2) t-\mathbb{E}_{x}\left[(f(Z(0)))^{+}\right]\right)-\mathbb{E}_{x}|Z(0)|
\end{aligned}
$$

for sufficiently large $t$. This implies

$$
\liminf _{t \rightarrow \infty} \frac{1}{t} \int_{0}^{t} \mathbb{E}_{x}|Z(s)| d s=\infty .
$$

Now we prove that

$$
\liminf _{t \rightarrow \infty} \frac{1}{t} \int_{0}^{t} \mathbb{E}_{x}[h(Z(s))] d s=\infty
$$

which implies $\mathrm{AC}(x, \varphi)=\infty$, thus proving the theorem.

For (4.10), by Assumption 1 (a) and (c), there exist constants $h_{1}>0$ and $c>0$ such that

$$
h^{\prime}(y) \geq h_{1} \text { for all } y \geq c \text { and } h^{\prime}(y) \leq-h_{1} \text { for all } y \leq-c .
$$


Because of (4.9), one of the following two equations holds:

$$
\begin{gathered}
\liminf _{t \rightarrow \infty} \frac{1}{t} \mathbb{E}_{x}\left(\int_{0}^{t} Z(s) 1_{\{Z(s) \geq c\}} d s\right)=\infty, \\
\liminf _{t \rightarrow \infty} \frac{1}{t} \mathbb{E}_{x}\left(\int_{0}^{t}|Z(s)| 1_{\{Z(s) \leq-c\}} d s\right)=\infty .
\end{gathered}
$$

Assume that (4.12) holds. Condition (4.11) implies

$$
h(-c)-h(y) \leq\left(-h_{1}\right)(-c-y) \quad \text { for } y \leq-c
$$

or

$$
h(y) \geq h_{1}|y|+h(-c)-c h_{1} \text { for } y \leq-c .
$$

Therefore,

$$
h(y) 1_{\{y \leq-c\}} \geq h_{1}|y| 1_{\{y \leq-c\}}-c h_{1} .
$$

Thus,

$$
\begin{aligned}
& \liminf _{t \rightarrow \infty} \frac{1}{t} \mathbb{E}_{x}\left(\int_{0}^{t} h(Z(s)) d s\right) \\
& \quad \geq \liminf _{t \rightarrow \infty} \frac{1}{t} \mathbb{E}_{x}\left(\int_{0}^{t} h(Z(s)) 1_{\{Z(s) \leq-c\}} d s\right) \\
& \quad \geq \liminf _{t \rightarrow \infty} \frac{1}{t}\left(\mathbb{E}_{x} \int_{0}^{t} h_{1}|Z(s)| 1_{\{Z(s) \leq-c\}} d s\right)-c h_{1} \\
& \quad=\infty
\end{aligned}
$$

which proves (4.10). Hence the theorem is proved for $K>0$ and $L>0$.

Case II: Assume that $K=0$ and $L=0$. Condition (4.2) leads to $f^{\prime}(u) \geq-k$ for all $u \in \mathbb{R}$ and condition (4.3) leads to $f^{\prime}(u) \leq \ell$ for all $u \in \mathbb{R}$. Because $f$ is continuous, $\Delta f(Z(s)) \neq 0$ implies $\Delta Z(s) \neq 0$. If $\Delta Z(s)>0$, (4.2) implies

$$
\Delta f(Z(s)) \geq-k \Delta Z(s) .
$$

If $\Delta Z(s)<0,(4.3)$ implies

$$
\Delta f(Z(s)) \geq \ell \Delta Z(s) .
$$

Thus, the last three terms in (4.4) are at least

$$
\begin{aligned}
& -k Y_{1}^{c}(t)-\ell Y_{2}^{c}(t)+\sum_{0 \leq s \leq t} \Delta f(Z(s)) \\
& \geq-k Y_{1}^{c}(t)-\ell Y_{2}^{c}(t)-k \sum_{\substack{0 \leq s \leq t \\
\Delta Z(s)>0}} \Delta Z(s)+\ell \sum_{\substack{0 \leq s \leq t \\
\Delta Z(s)<0}} \Delta Z(s)
\end{aligned}
$$




$$
\begin{aligned}
& =-k Y_{1}^{c}(t)-\ell Y_{2}^{c}(t)-k \sum_{0 \leq s \leq t} \Delta Y_{1}(s)-\ell \sum_{0 \leq s \leq t} \Delta Y_{2}(s) \\
& \geq-k Y_{1}(t)-\ell Y_{2}(t) .
\end{aligned}
$$

Therefore, (4.4) leads to

$$
\begin{gathered}
f(Z(t)) \geq f(Z(0-))+\gamma t-\int_{0}^{t} h(Z(s)) d s \\
\quad+\sigma \int_{0}^{t} f^{\prime}(Z(s)) d W(s)-k Y_{1}(t)-\ell Y_{2}(t)
\end{gathered}
$$

for $t \geq 0$. The remainder of the proof is identical to the case when $K>0$ and $L>0$.

Case III: Assume $K>0$ and $L=0$. Consider a feasible policy $\left(Y_{1}, Y_{2}\right)$ with a finite cost. The upward controls must be impulse controls and $Y_{1}(t)=$ $\sum_{n=0}^{N_{1}(t)} \xi_{1}(n)$. Condition (4.2) implies

$$
\sum_{\substack{0 \leq s \leq t \\ \Delta Z(s)>0}} \Delta f(Z(s)) \geq-\sum_{n=0}^{N_{1}(t)}\left(K+k \xi_{1}(n)\right) .
$$

and condition (4.3) implies

$$
-\ell Y_{2}^{c}(t)+\sum_{\substack{0 \leq s \leq t \\ \Delta Z(s)<0}} \Delta f(Z(s)) \geq-\ell Y_{2}(t) .
$$

Therefore, (4.4) leads to

$$
\begin{aligned}
f(Z(t)) \geq & f(Z(0-))+\gamma t-\int_{0}^{t} h(Z(s)) d s+\sigma \int_{0}^{t} f^{\prime}(Z(s)) d W(s) \\
& -\sum_{n=0}^{N_{1}(t)}\left(K+k \xi_{1}(n)\right)-\ell Y_{2}(t)
\end{aligned}
$$

for $t \geq 0$. The remainder of the proof is identical to the case when $K>0$ and $L>0$.

Case IV: Assume that $K=0$ and $L>0$. This case is analogous to the case when $K>0$ and $L=0$. Thus, the proof is omitted.

5. Impulse controls. We assume that $K>0$ and $L>0$. Therefore, we restrict our feasible policies to impulse controls as in (2.2) and (2.3). An impulse control band policy is defined by four parameters $d, D, U, u$, 
where $d<D<U<u$. Under the policy, when the inventory falls to $d$, the system instantaneously orders items to bring it up to level $D$; when the inventory rises to $u$, the system adjusts its inventory to bring it down to $U$. Given a control band policy $\varphi$, in Section 5.1 we provide a method for performance evaluation. As a by-product, we also obtain the relative value function associated with the control band policy. In Section 5.2, we first claim in Theorem 5.2 the existence of a solution to the free boundary problem with associated parameters $\left(d^{*}, D^{*}, U^{*}, u^{*}\right)$. Assuming Theorem 5.2 , we prove in Theorem 5.3 that the control band policy associated with $\left(d^{*}, D^{*}, U^{*}, u^{*}\right)$ is indeed optimal among all feasible policies. Section 5.3 describes the proof of Theorem 5.2.

5.1. Control band policies. Use $\{d, D, U, u\}$ to denote the control band policy $\varphi$ associated with parameters $d, D, U$ and $u$. Fix a control band policy $\varphi=\{d, D, U, u\}$ and an initial inventory level $Z(0-)=x$. The adjustment amount $\xi_{n}$ of the control band policy is given by

$$
\xi_{0}= \begin{cases}D-x & \text { if } x \leq d \\ 0 & \text { if } d<x<u \\ U-x & \text { if } x \geq u\end{cases}
$$

and for $n=1,2, \ldots$,

$$
\xi_{n}= \begin{cases}D-d & \text { if } Z\left(T_{n}-\right)=d \\ U-u & \text { if } Z\left(T_{n}-\right)=u\end{cases}
$$

where again $Z(t-)$ denotes the left limit at time $t, T_{0}=0$ and

$$
T_{n}=\inf \left\{t>T_{n-1}: Z(t) \in\{d, u\}\right\}
$$

is the $n$th adjustment time. (By convention, we assume $Z$ is right continuous having left limits.) Our first task is to find its long-run average cost. We first present the following theorem.

TheOrem 5.1. Assume that a control band policy $\varphi=\{d, D, U, u\}$ is fixed and that there exists a constant $\gamma$ and a twice continuously differentiable function $V:[d, u] \rightarrow \mathbb{R}$ that satisfies

$$
\Gamma V(x)+h(x)=\gamma, \quad d \leq x \leq u,
$$

with boundary conditions

$$
\begin{aligned}
& V(d)-V(D)=K+k(D-d), \\
& V(u)-V(U)=L+l(u-U),
\end{aligned}
$$


where $\Gamma$ is defined in (3.3). Then the average cost $A C(x, \varphi)$ is independent of the starting point $x \in \mathbb{R}$ and is given by $\gamma$ in (5.1).

Remark. Equation (5.1) is known as the Poisson equation. The solution $V$ is known as a relative value function associated with the control band policy $\varphi$. It is unique up to a constant. One can evaluate $\gamma$ from (5.1) by taking $x$ to be any value in $[d, u]$.

Proof. Consider control band policy $\varphi=\{d, D, U, u\}$. Let $V$ be a twice continuously differentiable function on $[d, u]$ that satisfies (5.1)-(5.3). Because $d \leq Z(t) \leq u$, by Lemma 3.1, we have

$$
\mathbb{E}_{x}[V(Z(t))]=\mathbb{E}_{x}[V(Z(0))]+\mathbb{E}_{x}\left[\int_{0}^{t} \Gamma V(Z(s)) d s\right]+\mathbb{E}_{x}\left[\sum_{n=1}^{N(t)} \theta_{n}\right],
$$

where $\theta_{n}=V\left(Z\left(T_{n}\right)\right)-V\left(Z\left(T_{n}-\right)\right)$. Boundary conditions (5.2) and (5.3) imply $\theta_{n}=V\left(Z\left(T_{n}\right)\right)-V\left(Z\left(T_{n}-\right)\right)=-\phi\left(\xi_{n}\right)$ for $n=1,2, \ldots$, where $\phi$ is defined in (4.5). Therefore,

$$
\begin{aligned}
\mathbb{E}_{x}[V(Z(t))]-\mathbb{E}_{x}[V(Z(0))] & =\mathbb{E}_{x}\left[\int_{0}^{t} \Gamma V(Z(s)) d s\right]+\mathbb{E}_{x}\left[\sum_{n=1}^{N(t)} \theta_{n}\right] \\
& =\gamma t-\mathbb{E}_{x}\left[\int_{0}^{t} h(Z(s)) d s\right]-\mathbb{E}_{x}\left[\sum_{n=1}^{N(t)} \phi\left(\xi_{n}\right)\right]
\end{aligned}
$$

Dividing both sides by $t$ and letting $t \rightarrow \infty$, we have $A C(x, \varphi)=\gamma$ because

$$
\lim _{t \rightarrow \infty} \frac{1}{t} \mathbb{E}_{x}[V(Z(t))]=0 \quad \text { and } \quad \mathbb{E}_{x}[V(Z(0))]=V\left(x+\xi_{0}\right) .
$$

We end this section by explicitly finding a solution $(V, \gamma)$ to (5.1)-(5.3). The solution $V$ is unique up to a constant. In the following proposition, let

$$
\lambda=2 \mu / \sigma^{2} \text {. }
$$

Proposition 1. Let $\varphi=\{d, D, U, u\}$ be a control band policy with

$$
d<D<U<u \text {. }
$$

Let $m \in \mathbb{R}$ be any fixed number. Define

$$
V(x)=\int_{m}^{x} g(y) d y \quad \text { for } x \in \mathbb{R}
$$


with

$$
g(x)=V^{\prime}(m) e^{\lambda(m-x)}+\gamma \frac{2}{\sigma^{2}} \int_{m}^{x} e^{\lambda(y-x)} d y-\frac{2}{\sigma^{2}} \int_{m}^{x} h(y) e^{\lambda(y-x)} d y,
$$

where

$$
\begin{aligned}
& \gamma=\frac{a_{1}\left(c_{2}+L+\ell(u-U)\right)+a_{2}\left(c_{1}+K+k(D-d)\right)}{a_{2} b_{1}+a_{1} b_{2}}, \\
& V^{\prime}(m)=\frac{b_{1}\left(c_{2}+L+\ell(u-U)\right)-b_{2}\left(c_{1}+K+k(D-d)\right)}{a_{2} b_{1}+a_{1} b_{2}} .
\end{aligned}
$$

Then $(V, \gamma)$ is a solution to (5.1)-(5.3). In (5.6) and (5.7), set

$$
\begin{aligned}
& a_{1}=\int_{d}^{D} e^{\lambda(m-x)} d x, \quad a_{2}=\int_{U}^{u} e^{\lambda(m-x)} d x \\
& b_{1}=-\frac{2}{\sigma^{2}} \int_{d}^{D} \int_{m}^{x} e^{\lambda(y-x)} d y d x, \quad b_{2}=\frac{2}{\sigma^{2}} \int_{U}^{u} \int_{m}^{x} e^{\lambda(y-x)} d y d x \\
& c_{1}=-\frac{2}{\sigma^{2}} \int_{d}^{D} \int_{m}^{x} h(y) e^{\lambda(y-x)} d y d x \\
& c_{2}=\frac{2}{\sigma^{2}} \int_{U}^{u} \int_{m}^{x} h(y) e^{\lambda(y-x)} d y d x
\end{aligned}
$$

Proof. Equation (5.1) is equivalent to

$$
\left(e^{\lambda x} V^{\prime}(x)\right)^{\prime}=\frac{2}{\sigma^{2}}(\gamma-h(x)) e^{\lambda x} .
$$

Integrating over $[m, x]$ on both sides, we have

$$
e^{\lambda x} V^{\prime}(x)=e^{\lambda m} V^{\prime}(m)+\gamma \frac{2}{\sigma^{2}} \int_{m}^{x} e^{\lambda y} d y-\frac{2}{\sigma^{2}} \int_{m}^{x} h(y) e^{\lambda y} d y
$$

or equivalently

$$
V^{\prime}(x)=e^{\lambda(m-x)} V^{\prime}(m)+\gamma \frac{2}{\sigma^{2}} \int_{m}^{x} e^{\lambda(y-x)} d y-\frac{2}{\sigma^{2}} \int_{m}^{x} h(y) e^{\lambda(y-x)} d y .
$$

Boundary conditions (5.2) and (5.3) become

$$
\begin{aligned}
& V^{\prime}(m) \int_{d}^{D} e^{\lambda(m-x)} d x+\gamma \frac{2}{\sigma^{2}} \int_{d}^{D} \int_{m}^{x} e^{\lambda(y-x)} d y d x \\
& =\frac{2}{\sigma^{2}} \int_{d}^{D} \int_{m}^{x} h(y) e^{\lambda(y-x)} d y d x-K-k(D-d), \\
& V^{\prime}(m) \int_{U}^{u} e^{\lambda(m-x)} d x+\gamma \frac{2}{\sigma^{2}} \int_{U}^{u} \int_{m}^{x} e^{\lambda(y-x)} d y d x \\
& =\frac{2}{\sigma^{2}} \int_{U}^{u} \int_{m}^{x} h(y) e^{\lambda(y-x)} d y d x+L+\ell(u-U) .
\end{aligned}
$$


Using the coefficients defined in (5.8)-(5.11), we now convert boundary conditions (5.12) and (5.13) into

$$
\begin{aligned}
& a_{1} V^{\prime}(m)-\gamma b_{1}=-\left(c_{1}+K+k(D-d)\right), \\
& a_{2} V^{\prime}(m)+\gamma b_{2}=c_{2}+L+\ell(u-U),
\end{aligned}
$$

from which we have the unique solution for $\gamma$ and $V^{\prime}(m)$ given in (5.6) and (5.7).

5.2. Optimal policy and optimal parameters. Theorem 4.1 suggests the following strategy to obtain an optimal policy. We hope that a control band policy is optimal. Therefore, the first task is to find an optimal policy among all control band policies. We denote this optimal control band policy by $\varphi^{*}=\left\{d^{*}, D^{*}, U^{*}, u^{*}\right\}$ with long-run average cost $\gamma^{*}$. We hope that $\gamma^{*}$ can be used as the constant in (4.1) of Theorem 4.1. To find the corresponding $f$ that, together with the $\gamma^{*}$, satisfies all the conditions of Theorem 4.1, we start with the relative value function $V(x)$ associated with the policy $\varphi^{*}$. This relative value function $V$ is defined on the finite interval $\left[d^{*}, u^{*}\right]$. We need to extend $V$ so that it is defined on the entire real line $\mathbb{R}$. Given that $V(x)$ is the relative value function, it is natural to extend it in the following way

$$
f(x)= \begin{cases}K+k\left(D^{*}-x\right)+V\left(D^{*}\right) & \text { for } x<d^{*} \\ V(x) & \text { for } x \in\left[d^{*}, u^{*}\right] \\ L+\ell\left(x-U^{*}\right)+V\left(U^{*}\right) & \text { for } x>u^{*}\end{cases}
$$

Boundary conditions (5.2) and (5.3) ensure the continuity of $f$ at $d^{*}$ and $u^{*}$. Therefore, $f \in C^{1}(\mathbb{R})$. However, we still need to determine the optimal parameters $\left(d^{*}, D^{*}, U^{*}, u^{*}\right)$. Now we provide an intuitive argument on the conditions that should be imposed on the optimal parameters. Since we wish $f \in C^{1}(\mathbb{R})$, we should have

$$
V^{\prime}\left(d^{*}\right)=-k, \quad V^{\prime}\left(u^{*}\right)=\ell
$$

Also, starting from $d^{*}$, the system should jump to a $D^{*}$ that minimizes

$$
p(D) \equiv K+k\left(D-d^{*}\right)+V(D) .
$$

Therefore, $p^{\prime}\left(D^{*}\right)=0$, namely,

$$
V^{\prime}\left(D^{*}\right)=-k
$$


A similar argument leads to

$$
V^{\prime}\left(U^{*}\right)=\ell
$$

Now, we first need to prove in Theorem 5.2 the existence of parameters $d^{*}, D^{*}, U^{*}$ and $u^{*}$ such that the relative value function $V$ corresponding to the control band policy $\varphi=\left\{d^{*}, D^{*}, U^{*}, u^{*}\right\}$ satisfies (5.1)-(5.3), and (5.15)(5.17). As part of the solution, we need to find the boundary points $d^{*}$, $D^{*}, U^{*}$ and $u^{*}$ from (5.1)-(5.3) and (5.15)-(5.17). These equations define a free boundary problem. We note that finding the solution to a free boundary problem is more difficult than finding the one to a boundary value problem. We then prove in Theorem 5.3 that the extension $f$ in $(5.14)$ and $\gamma^{*}=$ $\operatorname{AC}\left(\varphi^{*}, x\right)$ jointly satisfy all the conditions in Theorem 4.1 ; therefore, the control band policy $\varphi^{*}$ is optimal among all feasible policies.

To facilitate discussion, we assume that $\mu>0$. The statement and analysis for the cases $\mu<0$ and $\mu=0$ are analogous and are omitted.

To facilitate the presentation of Theorem 5.2, we first find a general solution $V$ to (5.1) without worrying about boundary conditions (5.2) and (5.3). Proposition 1 shows that such $V$ is given in the form

$$
V(x)=\int_{m}^{x} g(y) d y \quad \text { for } x \in\left[d^{*}, u^{*}\right]
$$

where $g$ is given by (5.5) and $m$ is some constant. Since the optimal boundary points $d^{*}, D^{*}, U^{*}, u^{*}$ are yet to be determined, the constant $\gamma$ on the right side of (5.1) is also yet to be determined. Differentiating both sides of (5.1) with respect to $x$, we have already shown that $V^{\prime}(x)=g(x)$ is a solution to

$$
\Gamma g(x)+h^{\prime}(x)=0 \quad \text { for all } x \in \mathbb{R} \backslash\{a\} .
$$

In (5.5), we fix $m=a$ and set $A=2 \gamma /\left(\lambda \sigma^{2}\right)$ and $B=A-V^{\prime}(m)$. Noting that $\lambda / \mu=\frac{2}{\sigma^{2}}$, we have $g(x)=g_{A, B}(x)$, where

$$
\begin{aligned}
g_{A, B}(x) & =A-B e^{-\lambda(x-a)}-(\lambda / \mu) \int_{a}^{x} h(y) e^{-\lambda(x-y)} d y \\
& =A-B e^{-\lambda(x-a)}-\frac{\lambda}{\mu} \int_{a}^{x} h(x-y+a) e^{-\lambda(y-a)} d y .
\end{aligned}
$$

To summarize, we have the following lemma.

Lemma 5.1. For each $A, B \in \mathbb{R}$, function $g(x)=g_{A, B}(x)$ is a solution to equation (5.19). 


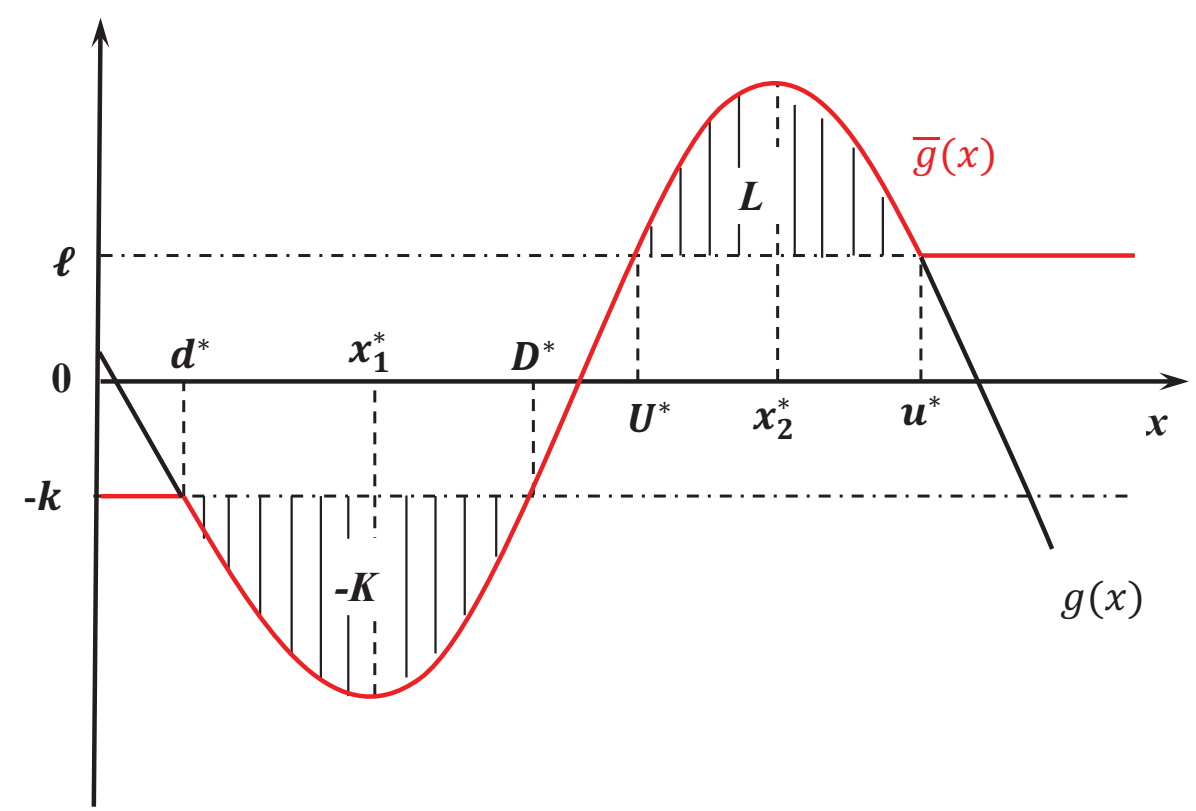

FIG 1. (a) There exist $x_{1}^{*}<x_{2}^{*}$ such that function $g$ decreases in $\left(-\infty, x_{1}^{*}\right)$, increases in $\left(x_{1}^{*}, x_{2}^{*}\right)$, and deceases again in $\left(x_{2}^{*}, \infty\right)$. Parameters $d^{*}, D^{*}, U^{*}$ and $u^{*}$ are determined by $g\left(d^{*}\right)=g\left(D^{*}\right)=-k, g\left(U^{*}\right)=g\left(u^{*}\right)=\ell$, the shaded area between $U^{*}$ and $u^{*}$ is $L$, and the shaded area between $d^{*}$ and $D^{*}$ is $K$. In the interval $\left[d^{*}, u^{*}\right], g$ is the derivative of the relative value function $V$ associated with control band policy $\varphi^{*}=\left\{d^{*}, D^{*}, U^{*}, u^{*}\right\}$.

(b) $\bar{g}(x)=-k$ for $x<d^{*}, \bar{g}(x)=g(x)$ for $d^{*} \leq x \leq u^{*}$ and $\bar{g}(x)=\ell$ for $x>u^{*}$.

The following theorem characterizes optimal parameters $\left(d^{*}, D^{*}, U^{*}, u^{*}\right)$ via solution $g=g_{A, B}$. Figure 1 depicts function $g$ as used in the theorem.

Theorem 5.2. Assume that holding cost function $h$ satisfies Assumption 1. There exist unique $A^{*}, B^{*}, x_{1}^{*}, x_{2}^{*}, d^{*}, D^{*}, U^{*}$ and $u^{*}$ with

$$
d^{*}<x_{1}^{*}<D^{*}<U^{*}<x_{2}^{*}<u^{*}
$$

such that the corresponding $g(x)=g_{A^{*}, B^{*}}(x)$ satisfies

$$
\begin{aligned}
& \int_{d^{*}}^{D^{*}}[g(x)+k] d x=-K, \\
& \int_{U^{*}}^{u^{*}}[g(x)-\ell] d x=L, \\
& g\left(d^{*}\right)=g\left(D^{*}\right)=-k, \\
& g\left(U^{*}\right)=g\left(u^{*}\right)=\ell .
\end{aligned}
$$


Furthermore, $g$ has a local minimum at $x_{1}^{*}<a$ and a local maximum at $x_{2}^{*}>a$. This function is decreasing on $\left(-\infty, x_{1}^{*}\right)$, increasing on $\left(x_{1}^{*}, x_{2}^{*}\right)$ and decreasing again on $\left(x_{2}^{*}, \infty\right)$.

If $g$ satisfies all conditions (5.19), (5.21)-(5.24) in Theorem 5.2, $V(x)$ in (5.18) clearly satisfies all conditions (5.1)-(5.3) and (5.15)-(5.17). The proof of Theorem 5.2 is long. Section 5.3 explains the proof.

THEOREM 5.3. Assume that holding cost function h satisfies Assumption 1. Let $d^{*}<D^{*}<U^{*}<u^{*}$, along with constants $A^{*}$ and $B^{*}$, be the unique solution in Theorem 5.2. Then control band policy $\varphi^{*}=\left\{d^{*}, D^{*}, U^{*}, u^{*}\right\}$ is optimal among all feasible policies.

Proof. Let $g(x)$ be the function in (5.20) with $A=A^{*}$ and $B=B^{*}$. Let

$$
\bar{g}(x)= \begin{cases}-k & \text { for } x<d^{*} \\ g(x) & \text { for } d^{*} \leq x \leq u^{*}, \\ \ell & \text { for } x>u^{*}\end{cases}
$$

see Figure 1. Conditions (5.23) and (5.24) ensure that $\bar{g}$ is $C(\mathbb{R})$. Define

$$
V(x)=\int_{d^{*}}^{x} \bar{g}(y) d y
$$

Let $\gamma^{*}$ be the long-run average cost under policy $\varphi^{*}$. We now show that $V$ and $\gamma^{*}$ satisfy all of the conditions in Theorem 4.1. Thus, Theorem 4.1 shows that the long-run average cost under any feasible policy is at least $\gamma^{*}$. Since $\gamma^{*}$ is the long-run average cost under the control band policy $\varphi^{*}$, $\gamma^{*}$ is the optimal cost and the control band policy $\varphi^{*}$ is optimal among all feasible policies.

First, $V(x)$ is in $C^{2}\left(\left(d^{*}, u^{*}\right)\right)$. Condition (5.21) implies

$$
V\left(d^{*}\right)-V\left(D^{*}\right)=K+k\left(D^{*}-d^{*}\right)
$$

and (5.22) implies

$$
V\left(u^{*}\right)-V\left(U^{*}\right)=L+\ell\left(u^{*}-U^{*}\right) .
$$

Equation (5.19) implies that $V$ satisfies

$$
\Gamma V(x)+h(x)=\text { constant } \quad \text { for } x \in\left(d^{*}, u^{*}\right) .
$$

According to Theorem 5.1, the constant must be the long-run average cost $\gamma^{*}$ under control band policy $\varphi^{*}$. 
Now we show that $V(x)$ satisfies the remaining conditions in Theorem 4.1. Conditions (5.23) and (5.24) imply that truncated function $\bar{g}$ is continuous in $\mathbb{R}$. Therefore, $V \in C^{1}(\mathbb{R})$. Clearly, $V^{\prime \prime}(x)=0$ for $x \notin\left[d^{*}, u^{*}\right]$, and $V^{\prime \prime}(x)=$ $g^{\prime}(x)$ for $x \in\left(d^{*}, u^{*}\right)$. Let

$$
M=\sup _{x \in\left[d^{*}, u^{*}\right]}|g(x)|
$$

We have $\left|V^{\prime}(x)\right| \leq M$ for all $x \in \mathbb{R}$. Because

$$
\Gamma V(x)+h(x)=\gamma^{*} \quad \text { for } x \in\left(d^{*}, u^{*}\right),
$$

(4.1) is satisfied for $x \in\left(d^{*}, u^{*}\right)$. In particular

$$
\frac{1}{2} \sigma^{2} g^{\prime}\left(d^{*}\right)+\mu g\left(d^{*}\right)+h\left(d^{*}\right)=\gamma^{*}
$$

and

$$
\frac{1}{2} \sigma^{2} g^{\prime}\left(u^{*}\right)+\mu g\left(u^{*}\right)+h\left(u^{*}\right)=\gamma^{*} .
$$

From Theorem 5.2, it follows that $d^{*}<x_{1}^{*}<a<x_{2}^{*}<u^{*}, g^{\prime}\left(d^{*}\right) \leq 0$ and $g^{\prime}\left(u^{*}\right) \leq 0$ (see Figure 1). Thus, we have $\mu g\left(d^{*}\right)+h\left(d^{*}\right) \geq \gamma^{*}$ and $\mu g\left(u^{*}\right)+h\left(u^{*}\right) \geq \gamma^{*}$. Now, for $x<d^{*}, \Gamma V(x)+h(x)=\mu(-k)+h(x) \geq$ $\mu g\left(d^{*}\right)+h\left(d^{*}\right) \geq \gamma^{*}$. Similarly, for $x>u^{*}, \Gamma V(x)+h(x)=\mu(\ell)+h(x) \geq$ $\mu g\left(u^{*}\right)+h\left(u^{*}\right) \geq \gamma^{*}$.

Now we verify that $V$ satisfies (4.2). Let $x, y \in \mathbb{R}$ with $y<x$. Then,

$$
\begin{aligned}
V(x)-V(y)+k(x-y) & =\int_{y}^{x}[\bar{g}(z)+k] d z \\
& \geq \int_{\left(y \vee d^{*}\right) \wedge D^{*}}^{\left(x \wedge D^{*}\right) \vee d^{*}}[\bar{g}(z)+k] d z \\
& \geq \int_{d^{*}}^{D^{*}}[\bar{g}(z)+k] d z \\
& =-K
\end{aligned}
$$

where the first inequality follows from the fact that $\bar{g}(z)=g(z)=-k$ for $z \leq d^{*}$ and $\bar{g}(z)=g(z) \geq-k$ for $D^{*}<z<u^{*}$ and $\bar{g}(z)=\ell \geq-k$ for $z \geq u^{*}$, and the second inequality follows from the fact that $\bar{g}(z)=g(z) \leq-k$ for $z \in\left[d^{*}, D^{*}\right]$; see Figure 1. Thus (4.2) is proved.

It remains to verify that $V$ satisfies (4.3). For $x, y \in \mathbb{R}$ with $y>x$.

$$
V(y)-V(x)-\ell(y-x)=\int_{x}^{y}[\bar{g}(z)-\ell] d z
$$




$$
\begin{aligned}
& \leq \int_{\left(x \vee U^{*}\right) \wedge u^{*}}^{\left(y \wedge u^{*}\right) \vee U^{*}}[\bar{g}(z)-\ell] d z \\
& \leq \int_{U^{*}}^{u^{*}}[\bar{g}(z)-\ell] d z \\
& =L,
\end{aligned}
$$

proving (4.3).

5.3. Optimal control band parameters. This section explains the proof of Theorem 5.2. Throughout this section, we assume that $\mu>0$ and that the holding cost function $h$ satisfies Assumption 1. Recall that the $\lambda$ is defined in (5.4) and that $g(x)=g_{A, B}(x)$, defined in (5.20), depends on two parameters $A$ and $B$. To prove the theorem, it suffices to prove that there exists $\left(A^{*}, B^{*}\right)$ so that $g(x)=g_{A^{*}, B^{*}}(x)$, together with some constants $x_{1}^{*}, x_{2}^{*}, d^{*}, D^{*}, U^{*}$, $u^{*}$, satisfies (5.21)-(5.24) and the desired monotonicity properties of $g(x)$ as stated in the theorem. Recall that Figure 1 illustrates the conditions and properties that $g_{A^{*}, B^{*}}(x)$ must satisfy. To prove the existence of $\left(A^{*}, B^{*}\right)$, we start with a series of lemmas. Each lemma progressively narrows the search range of $(A, B)$ so that $g_{A, B}(x)$ satisfies a subset of the conditions in Theorem 5.2.

Before stating and proving these lemmas, we describe them briefly. Let $\bar{B}>0$ be the constant defined in (5.25) below. In Lemma 5.2, we show that for each $B \in(0, \bar{B})$, the function $g_{A, B}(x)$ has a unique local minimum point $x_{1}(B)$ and a unique local maximum point $x_{2}(B)$. The function $g_{A, B}(x)$ decreases in $\left(-\infty, x_{1}(B)\right)$, increases in $\left(x_{1}(B), x_{2}(B)\right)$, and decreases again in $\left(x_{2}(B), \infty\right)$; see Figure 1. In Lemma 5.3, we show that $x_{i}(B)$ is monotone and continuous in $B, i=1,2$.

In Lemma 5.4, we first show that the distance between the local maximum and the local minimum of $g_{A, B}$, denoted as $\widetilde{g}(B)=g_{A, B}\left(x_{2}(B)\right)-$ $g_{A, B}\left(x_{1}(B)\right)$, is continuous and strictly increasing in $B$, and there exists a unique $\underline{B}_{1} \in(0, \bar{B})$ such that $\widetilde{g}\left(\underline{B}_{1}\right)=k+\ell$. Furthermore, for each fixed $B \in\left(\underline{B}_{1}, \bar{B}\right)$, we identify an interval of $A,(\underline{A}(B), \bar{A}(B)]$, in which $g_{A, B}\left(x_{1}(B)\right) \leq-k$ and $g_{A, B}\left(x_{2}(B)\right)>\ell$. As a consequence, in Lemma 5.5, we show that for $B \in\left(\underline{B}_{1}, \bar{B}\right)$ and $A \in(\underline{A}(B), \bar{A}(B)]$, curve $y=g_{A, B}(x)$ has exactly two intersections, denoted by $U(A, B)$ and $u(A, B)$, with curve $y=\ell$, and therefore condition (5.24) is always satisfied for $U(A, B)$ and $u(A, B)$; see Figure 1 .

In Lemmas 5.6-5.8, we identify a value $\underline{B}_{2} \in\left(\underline{B}_{1}, \bar{B}\right)$ and then prove that for $B \in\left(\underline{B}_{2}, \bar{B}\right)$ there exists a unique $A^{*}(B) \in(\underline{A}(B), \bar{A}(B)]$ such that the shaded area in Figure 1, bounded by curve $g_{A^{*}(B), B}(x)$ and line $y=\ell$, is 
equal to $L$. Thus, $g_{A, B}(x)$ satisfies condition (5.22). Finally, in Lemma 5.9, we show that there exists a unique $B^{*} \in\left(\underline{B}_{2}, \bar{B}\right)$ such that the shaded area below line $y=-k$ is equal to $-K$. Curve $y=g_{A^{*}\left(B^{*}\right), B^{*}}(x)$ has exactly two intersections, denoted by $d^{*}$ and $D^{*}$, with curve $y=-k$. Thus, conditions (5.21) and (5.23) are satisfied.

These lemmas show that by choosing $x_{1}^{*}=x_{1}\left(B^{*}\right), x_{2}^{*}=x_{2}\left(B^{*}\right), A^{*}=$ $A^{*}\left(B^{*}\right), U^{*}=U\left(A^{*}, B^{*}\right)$ and $u^{*}=u\left(A^{*}, B^{*}\right)$, function $g_{A^{*}, B^{*}}(x)$, together with $x_{1}^{*}, x_{2}^{*}, d^{*}, D^{*}, U^{*}$ and $u^{*}$, satisfies conditions (5.21)-(5.24) and associated monotonicity properties. Therefore, Theorem 5.2 is proved.

Define

$$
\bar{B}=-\frac{1}{\mu} \int_{-\infty}^{a} h^{\prime}(y) e^{\lambda(y-a)} d y .
$$

Because $h^{\prime}(x)<0$ for $x<a, \bar{B}>0$. For $A, B \in \mathbb{R}$, recall the function $g_{A, B}$ defined in (5.20). We sometimes use the fact that

$$
g_{A, B}(x)=A+g_{0, B}(x) \quad \text { for } x \in \mathbb{R} .
$$

When the context is clear, we simply use $g$ to denote $g_{A, B}$. For the following lemma, see Figure 1.

Lemma 5.2. (a) For any $A \in \mathbb{R}$ and for each fixed $B \in(0, \bar{B}), g_{A, B}$ attains a unique minimum in $(-\infty, a)$ at $x_{1}=x_{1}(B) \in(-\infty, a)$. Function $g_{A, B}$ attains a unique maximum in $(a, \infty)$ at $x_{2}=x_{2}(B) \in(a, \infty)$. Both $x_{1}(B)$ and $x_{2}(B)$ are independent of $A$.

(b) For each fixed $B \in(0, \bar{B})$, the local minimizer $x_{1}=x_{1}(B)$ is the unique solution in $(-\infty, a)$ to

$$
B-\frac{1}{\mu} \int_{a}^{x} h^{\prime}(y) e^{\lambda(y-a)} d y=0 .
$$

The local maximizer $x_{2}=x_{2}(B)$ is the unique solution in $(a, \infty)$ to (5.27).

(c) For each $B \in(0, \bar{B}), g_{A, B}^{\prime}(x)<0$ for $x \in\left(-\infty, x_{1}(B)\right), g_{A, B}^{\prime}(x)>0$ for $x \in\left(x_{1}(B), x_{2}(B)\right)$, and $g_{A, B}^{\prime}(x)<0$ for $x \in\left(x_{2}(B), \infty\right)$.

Proof. Differentiating $g(x)=g_{A, B}(x)$ in (5.20) and noting $h(a)=0$, we have

$$
\begin{aligned}
g^{\prime}(x) & =\lambda B e^{-\lambda(x-a)}-\frac{\lambda}{\mu} \int_{a}^{x} h^{\prime}(x-y+a) e^{-\lambda(y-a)} d y \\
& =\lambda\left(B-\frac{1}{\mu} \int_{a}^{x} h^{\prime}(y) e^{\lambda(y-a)} d y\right) e^{-\lambda(x-a)} \\
& =\lambda F_{1}(B, x) e^{-\lambda(x-a)},
\end{aligned}
$$


where, for $x \in \mathbb{R}$,

$$
F_{1}(B, x)=B-\frac{1}{\mu} \int_{a}^{x} h^{\prime}(y) e^{\lambda(y-a)} d y .
$$

Clearly $g^{\prime}(x)=0$ if and only if $F_{1}(B, x)=0$. Because

$$
\frac{\partial}{\partial x} F_{1}(B, x)=-\frac{1}{\mu} h^{\prime}(x) e^{\lambda(x-a)}
$$

and $h^{\prime}(x)<0$ for $x<a$ and $h^{\prime}(x)>0$ for $x>a, F_{1}(B, x)$ increases in $x<a$ and decreases in $x>a$. For $B>0$,

$$
F_{1}(B, a)=B>0 .
$$

For any $B \in(0, \bar{B})$,

$$
\lim _{x \downarrow-\infty} F_{1}(B, x)=B-\bar{B}<0 .
$$

Therefore, there exists a unique $x_{1}=x_{1}(B) \in(-\infty, a)$ such that $F_{1}\left(B, x_{1}\right)=$ 0 or equivalently $g^{\prime}\left(x_{1}\right)=0$. Also, for any fixed $B$,

$$
\lim _{x \uparrow+\infty} F_{1}(B, x)=-\infty .
$$

Therefore, for any $B>0$, there exists a unique $x_{2}=x_{2}(B) \in(a, \infty)$ such that $F_{1}\left(B, x_{2}\right)=0$ or equivalently $g^{\prime}\left(x_{2}\right)=0$. For $B \in(0, \bar{B})$, it is clear that

$$
\begin{aligned}
& g^{\prime}(x)<0 \text { for } x \in\left(-\infty, x_{1}\right), \quad g^{\prime}(x)>0 \text { for } x \in\left(x_{1}, x_{2}\right) \quad \text { and } \\
& g^{\prime}(x)<0 \text { for } x \in\left(x_{2}, \infty\right) .
\end{aligned}
$$

Thus the lemma is proved.

Remark. The local maximizer $x_{2}(B)$ is well defined for all $B \in(0, \infty)$, whereas the local minimizer $x_{1}(B)$ is defined only for $B \in(0, \bar{B})$.

Lemma 5.3. (a) The local minimizer $x_{1}(B)$ is continuous and strictly decreasing in $B \in(0, \bar{B})$. The local maximizer $x_{2}(B)$ is continuous and strictly increasing in $B \in(0, \infty)$. Furthermore,

$$
\lim _{B \downarrow 0} x_{i}(B)=a \quad i=1,2
$$

and

$$
\lim _{B \uparrow \bar{B}} x_{1}(B)=-\infty, \quad \lim _{B \uparrow \bar{B}} x_{2}(B)=x_{2}(\bar{B}) \in(a, \infty) .
$$


(b) For each $B \in(0, \bar{B})$,

$$
g_{A, B}\left(x_{i}(B)\right)=A-\frac{1}{\mu} h\left(x_{i}(B)\right) \quad \text { for } i=1,2 .
$$

Proof. (a) Recall the function $F_{1}$ defined in (5.28). Obviously, $F_{1}, \frac{\partial F_{1}}{\partial B}$, $\frac{\partial F_{1}}{\partial x}$ are continuous, and $\frac{\partial F_{1}}{\partial x}$ is given in (5.29). Therefore,

$$
\frac{\partial F_{1}}{\partial x}>0 \quad \text { for } x \in(-\infty, a)
$$

where we use the fact that $h^{\prime}(x)<0$ for $x \in(-\infty, a)$. Using the Implicit Function Theorem, $x_{1}(B)$ is continuously differentiable in $B \in(0, \bar{B})$, and

$$
\frac{d x_{1}(B)}{d B}=\frac{\mu}{h^{\prime}\left(x_{1}(B)\right) e^{\lambda\left(x_{1}(B)-a\right)}}<0 .
$$

Thus, $x_{1}(B)$ is strictly decreasing in $B \in(0, \bar{B})$. Similarly,

$$
\frac{d x_{2}(B)}{d B}=\frac{\mu}{h^{\prime}\left(x_{2}(B)\right) e^{\lambda\left(x_{2}(B)-a\right)}}>0
$$

proves that $x_{2}(B)$ is continuously differentiable and strictly increasing in $B \in(0, \infty)$. The limits in $(5.30)$ and $(5.31)$ can be proved easily following the definition of $x_{1}(B)$ and $x_{2}(B)$.

(b) From (5.20) and (5.27), for $i=1,2$, we have

$$
\begin{aligned}
& g_{A, B}\left(x_{i}(B)\right) \\
&=A-B e^{-\lambda\left(x_{i}(B)-a\right)}-\frac{\lambda}{\mu} \int_{a}^{x_{i}(B)} h\left(x_{i}(B)-y+a\right) e^{-\lambda(y-a)} d y \\
&=A-\frac{1}{\mu} \int_{a}^{x_{i}(B)} h^{\prime}\left(x_{i}(B)-y+a\right) e^{-\lambda(y-a)} d y \\
& \quad-\frac{\lambda}{\mu} \int_{a}^{x_{i}(B)} h\left(x_{i}(B)-y+a\right) e^{-\lambda(y-a)} d y \\
&= A-\frac{\lambda}{\mu} \int_{a}^{x_{i}(B)} h\left(x_{i}(B)-y+a\right) e^{-\lambda(y-a)} d y \\
&+\frac{1}{\mu}\left[\left.h\left(x_{i}(B)-y+a\right) e^{-\lambda(y-a)}\right|_{a} ^{x_{i}(B)}\right. \\
&\left.+\lambda \int_{a}^{x_{i}(B)} h\left(x_{i}(B)-y+a\right) e^{-\lambda(y-a)} d y\right] \\
&= A-\frac{1}{\mu} h\left(x_{i}(B)\right),
\end{aligned}
$$

thus proving (5.32). 
In the following lemma, for each $B \in(0, \infty)$, we set

$$
\underline{A}(B)=\ell-g_{0, B}\left(x_{2}(B)\right) .
$$

For any $B \in(0, \infty)$, following (5.26) and (5.32), we have

$$
g_{A, B}\left(x_{2}(B)\right) \geq \ell
$$

for any $A \geq \underline{A}(B)$. Similarly, for any $B \in(0, \bar{B})$, we define

$$
\bar{A}(B)=-k-g_{0, B}\left(x_{1}(B)\right) .
$$

Following (5.26) and (5.32), we have

$$
g_{A, B}\left(x_{1}(B)\right) \leq-k
$$

for any $A \leq \bar{A}(B)$. Our next lemma determines when $\underline{A}(B)<\bar{A}(B)$.

Lemma 5.4. For each $B \in(0, \bar{B})$, let

$$
\tilde{g}(B)=g_{A, B}\left(x_{2}(B)\right)-g_{A, B}\left(x_{1}(B)\right)
$$

be the distance between the local maximum and the local minimum. Then $\tilde{g}(B)$ is independent of $A$. Function $\tilde{g}(B)$ is continuous and strictly increasing in $B \in(0, \bar{B})$ with

$$
\lim _{B \downarrow 0} \widetilde{g}(B)=0 \quad \text { and } \quad \lim _{B \uparrow \bar{B}} \widetilde{g}(B)=+\infty .
$$

Thus, there exists a unique $\underline{B}_{1} \in(0, \bar{B})$ such that

$$
\tilde{g}\left(\underline{B}_{1}\right)=k+\ell \text {. }
$$

For each $B \in\left(\underline{B}_{1}, \bar{B}\right)$,

$$
\underline{A}(B)<\bar{A}(B) \text {. }
$$

Proof. By (5.26), $\tilde{g}(B)=g_{0, B}\left(x_{2}(B)\right)-g_{0, B}\left(x_{1}(B)\right)$. Thus, $\tilde{g}(B)$ is independent of $A$. From (5.33) and (5.34), it follows that for $B \in(0, \bar{B})$,

$$
\begin{aligned}
\frac{d \tilde{g}(B)}{d B} & =-\frac{1}{\mu}\left[h^{\prime}\left(x_{2}(B)\right) \frac{d x_{2}(B)}{d B}-h^{\prime}\left(x_{1}(B)\right) \frac{d x_{1}(B)}{d B}\right] \\
& =-e^{-\lambda\left(x_{2}(B)-a\right)}+e^{-\lambda\left(x_{1}(B)-a\right)} \\
& >0 .
\end{aligned}
$$

Thus $\tilde{g}(B)$ is strictly increasing. The limit (5.38) follows from (5.30) and (5.31). The existence of unique $\underline{B}_{1}$ satisfying (5.39) follows from (5.38), the continuity and monotonicity of $\tilde{g}$. Inequality (5.40) follows from the definition of $\underline{B}_{1}$ and the fact that $\bar{A}(B)-\underline{A}(B)=\tilde{g}(B)-(\ell+k)$. 
Lemma 5.5. (a) For each $B \in\left(\underline{B}_{1}, \bar{B}\right)$ and each $A \in(\underline{A}(B), \bar{A}(B)]$, there exist unique $U(A, B)$ and $u(A, B)$ with

$$
x_{1}(B)<U(A, B)<x_{2}(B)<u(A, B)
$$

such that

$$
\begin{aligned}
& g_{A, B}(U(A, B))=g_{A, B}(u(A, B))=\ell, \\
& g_{A, B}^{\prime}(U(A, B))>0, \quad g_{A, B}^{\prime}(u(A, B))<0, \\
& g_{A, B}\left(x_{1}(B)\right) \leq-k .
\end{aligned}
$$

(b) For each fixed $B \in\left(\underline{B}_{1}, \bar{B}\right), U(A, B)$ and $u(A, B)$ are continuously differentiable function in $A \in(\underline{A}(B), \bar{A}(B))$. Function $U(A, B)$ is decreasing in $A$ and function $u(A, B)$ is increasing in $A$.

Proof. (a) For each $B \in\left(\underline{B}_{1}, \bar{B}\right)$ and each $A \in(\underline{A}(B), \bar{A}(B)]$, we have $g_{A, B}\left(x_{2}(B)\right)>\ell$ and $g_{A, B}\left(x_{1}(B)\right) \leq-k$. Thus, there are unique $U(A, B)$ and $u(A, B)$ that satisfy (5.43)-(5.44). When $A \in(\underline{A}(B), \bar{A}(B)]$, inequality (5.45) holds. This inequality implies $U(A, B)>x_{1}(B)$, which in turn implies that inequality (5.42) holds.

(b) Using the Implicit Function Theorem, we have

$$
\begin{aligned}
& \frac{\partial}{\partial A} U(A, B)=-\frac{1}{g_{A, B}^{\prime}(U(A, B))}<0, \\
& \frac{\partial}{\partial A} u(A, B)=-\frac{1}{g_{A, B}^{\prime}(u(A, B))}>0 .
\end{aligned}
$$

This proves part (b) of the lemma.

Fix a $B \in\left(\underline{B}_{1}, \bar{B}\right)$. For $A \in(\underline{A}(B), \bar{A}(B)]$ let

$$
\Lambda_{2}(A, B)=\int_{u(A, B)}^{U(A, B)}\left[g_{A, B}(x)-\ell\right] d x .
$$

We would like to show that there exists a unique $A^{*}(B) \in(\underline{A}(B), \bar{A}(B)]$ such that

$$
\Lambda_{2}\left(A^{*}(B), B\right)=L \text {. }
$$

Lemma 5.6. Fix a $B \in\left(\underline{B}_{1}, \bar{B}\right)$. Function $\Lambda_{2}(A, B)$ is continuous and strictly increasing in $A \in(\underline{A}(B), \bar{A}(B)]$. Furthermore

$$
\lim _{A \downarrow \underline{A}(B)} \Lambda_{2}(A, B)=0 .
$$


Proof. By the Implicit Function Theorem, we have

$$
\begin{aligned}
\frac{\partial \Lambda_{2}(A, B)}{\partial A}= & \frac{\partial u(A, B)}{\partial A}\left[g_{A, B}(u(A, B)-l)\right] \\
& -\frac{\partial U(A, B)}{\partial A}\left[g_{A, B}(U(A, B)-l)\right]+\int_{U(A, B)}^{u(A, B)} 1 d x \\
= & u(A, B)-U(A, B) \\
> & 0 .
\end{aligned}
$$

Therefore $\Lambda_{2}(A, B)$ is strictly increasing in $A \in(\underline{A}(B), \bar{A}(B)]$.

We observe

$$
\lim _{A \downarrow \underline{A}(B)} g_{A, B}\left(x_{2}(B)\right)=\lim _{A \downarrow \underline{A}(B)}\left[A-\frac{1}{\mu} h\left(x_{2}(B)\right)\right]=l .
$$

By the definitions of $U(A, B)$ and $u(A, B)$, we have

$$
\lim _{A \downarrow \underline{A}(B)} U(A, B)=\lim _{A \downarrow \underline{A}(B)} u(A, B)=x_{2}(B),
$$

which proves (5.46).

Lemma 5.7. Function $\Lambda_{2}(\bar{A}(B), B)$ is continuous and strictly increasing in $B \in\left(\underline{B}_{1}, \bar{B}\right)$. Furthermore,

$$
\lim _{B \downarrow \underline{B}_{1}} \Lambda_{2}(\bar{A}(B), B)=0 \quad \text { and } \quad \lim _{B \uparrow \bar{B}} \Lambda_{2}(\bar{A}(B), B)=\infty .
$$

Therefore, there exists a unique $\underline{B}_{2} \in\left(\underline{B}_{1}, \bar{B}\right)$ such that

$$
\Lambda_{2}\left(\bar{A}\left(\underline{B}_{2}\right), \underline{B}_{2}\right)=L \quad \text { and } \quad \Lambda_{2}(\bar{A}(B), B)>L \quad \text { for } B \in\left(\underline{B}_{2}, \bar{B}\right) .
$$

Proof. We first check that $\Lambda_{2}(\bar{A}(B), B)$ is strictly increasing in $B \in$ $\left(\underline{B}_{1}, \bar{B}\right)$. From (5.33) and the definition of $\bar{A}(B)$ in (5.36), it follows that

$$
\frac{d \bar{A}(B)}{d B}=\frac{1}{\mu} h^{\prime}\left(x_{1}(B)\right) \frac{d x_{1}(B)}{d B}=e^{-\lambda\left(x_{1}(B)-a\right)},
$$

which implies

$$
\begin{gathered}
\frac{d \Lambda_{2}(\bar{A}(B), B)}{d B}=\frac{\partial u(\bar{A}(B), B)}{\partial B}\left[g_{\bar{A}(B), B}(u(A, B)-l)\right] \\
+\frac{\partial u(\bar{A}(B), B)}{\partial A} \frac{d \bar{A}(B)}{d B}\left[g_{\bar{A}(B), B}(u(A, B)-l)\right]
\end{gathered}
$$




$$
\begin{aligned}
& -\frac{\partial U(\bar{A}(B), B)}{\partial B}\left[g_{\bar{A}(B), B}(U(A, B)-l)\right] \\
& -\frac{\partial U(\bar{A}(B), B)}{\partial A} \frac{d \bar{A}(B)}{d B}\left[g_{\bar{A}(B), B}(U(A, B)-l)\right] \\
& \quad+\int_{U(A, B)}^{u(A, B)}\left[-e^{-\lambda(x-a)}+\frac{d \bar{A}(B)}{d B}\right] d x \\
& =\int_{U(A, B)}^{u(A, B)}\left[-e^{-\lambda(x-a)}+e^{-\lambda\left(x_{1}(B)-a\right)}\right] d x \\
& >0
\end{aligned}
$$

where the last inequality is due to $x_{1}(B)<U(A, B)<u(A, B)$ for $B \in$ $\left(\underline{B}_{1}, \bar{B}\right)$. Thus, we have proved that $\Lambda_{2}(\bar{A}(B), B)$ is strictly increasing in $B \in\left(\underline{B}_{1}, \bar{B}\right)$.

Because $\tilde{g}\left(\underline{B}_{1}\right)=k+\ell$,

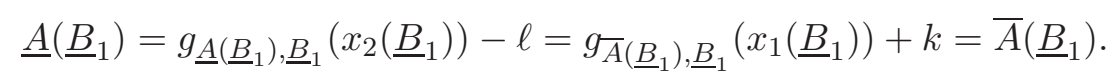

Thus,

$$
\lim _{B \downarrow \underline{B}_{1}} U(\bar{A}(B), B)=\lim _{B \downarrow \underline{B}_{1}} u(\bar{A}(B), B)=x_{2}\left(\underline{B}_{1}\right) .
$$

It follows that

$$
\lim _{B \downarrow \underline{B}_{1}} \Lambda_{2}(\bar{A}(B), B)=0 .
$$

We now show that

$$
\lim _{B \uparrow \bar{B}} \Lambda_{2}(\bar{A}(B), B)=\infty .
$$

It is clear that (5.49), (5.50) and the monotonicity imply the existence of a unique $\underline{B}_{2} \in\left(\underline{B}_{1}, \bar{B}\right)$ that satisfies $(5.47)$.

To prove $(5.50)$, we check that

$$
\begin{aligned}
& \frac{d U(\bar{A}(B), B)}{d B}=\frac{e^{-\lambda(U(\bar{A}(B), B)-a)}-e^{\left.-\lambda\left(x_{1}(B)\right)-a\right)}}{g_{\bar{A}(B), B}^{\prime}(U(\bar{A}(B), B))}<0, \\
& \frac{d u(\bar{A}(B), B)}{d B}=\frac{e^{-\lambda(u(\bar{A}(B), B)-a)}-e^{\left.-\lambda\left(x_{1}(B)\right)-a\right)}}{g_{\bar{A}(B), B}^{\prime}(u(\bar{A}(B)))}>0 .
\end{aligned}
$$

Therefore, $U(\bar{A}(B), B)$ decreases in $B$ and $u(\bar{A}(B), B)$ increases in $B$. Thus,

$$
U(\bar{A}(B), B) \leq U\left(\bar{A}\left(\underline{B}_{1}\right), \underline{B}_{1}\right) \quad \text { and } \quad u(\bar{A}(B), B) \geq u\left(\bar{A}\left(\underline{B}_{1}\right), \underline{B}_{1}\right)
$$


as $B \uparrow \bar{B}$. Therefore, noting that $g_{\bar{A}(B), B}(x)-l \geq 0$ for $x \in(U(\bar{A}(B), B)$, $u(\bar{A}(B), B))$, we have

$$
\begin{aligned}
\lim _{B \uparrow \bar{B}} \Lambda_{2}(\bar{A}(B), B)= & \lim _{B \uparrow \bar{B}} \int_{U(\bar{A}(B), B)}^{u(\bar{A}(B), B)}\left[g_{\bar{A}(B), B}(x)-l\right] d x \\
\geq & \lim _{B \uparrow \bar{B}} \int_{U\left(\bar{A}\left(\underline{B}_{1}\right), \underline{B}_{1}\right)}^{\left.u\left(\underline{B}_{1}\right), \underline{B}_{1}\right)}\left[g_{\bar{A}(B), B}(x)-l\right] d x \\
= & \lim _{B \uparrow \bar{B}} \int_{U\left(\bar{A}\left(\underline{B}_{1}\right), \underline{B}_{1}\right)}^{u\left(\bar{A}\left(\underline{B}_{1}\right), \underline{B}_{1}\right)}\left[\bar{A}(B)+g_{0, B}(x)-l\right] d x \\
= & \left(U\left(\bar{A}\left(\underline{B}_{1}\right), \underline{B}_{1}\right)-u\left(\bar{A}\left(\underline{B}_{1}\right), \underline{B}_{1}\right)\right) \lim _{B \uparrow \bar{B}} \bar{A}(B) \\
& +\int_{U\left(\bar{A}\left(\underline{B}_{1}\right), \underline{B}_{1}\right)}^{\left.u\left(\underline{B}_{1}\right), \underline{B}_{1}\right)}\left[g_{0, \bar{B}}(x)-l\right] d x \\
= & \infty,
\end{aligned}
$$

where we have used the fact that

$$
\lim _{B \uparrow \bar{B}} \bar{A}(B)=\lim _{B \uparrow \bar{B}} h_{1}\left(x_{1}(B)\right) / \mu-k=\infty .
$$

Lemma 5.6 and the inequality in (5.47) immediately imply the following lemma.

Lemma 5.8. For each $B \in\left[\underline{B}_{2}, \bar{B}\right)$, there exists a unique $A^{*}(B) \in$ $(\underline{A}(B), \bar{A}(B)]$ such that

$$
\Lambda_{2}\left(A^{*}(B), B\right)=L
$$

Finally, we prove the following lemma, which in turn proves Theorem 5.2.

Lemma 5.9. There exist unique $B^{*} \in\left(\underline{B}_{2}, \bar{B}\right), d^{*}$ and $D^{*}$ that satisfy

$$
\begin{aligned}
& d^{*}<x_{1}\left(B^{*}\right)<D^{*}<U\left(A^{*}\left(B^{*}\right), B^{*}\right), \\
& g_{A^{*}\left(B^{*}\right), B^{*}}\left(d^{*}\right)=g_{A^{*}\left(B^{*}\right), B^{*}}\left(D^{*}\right)=-k, \\
& g_{A^{*}\left(B^{*}\right), B^{*}}^{\prime}\left(d^{*}\right)<0, g_{A^{*}\left(B^{*}\right), B^{*}}^{\prime}\left(D^{*}\right)>0, \\
& \int_{d^{*}}^{D^{*}}\left[g_{A^{*}\left(B^{*}\right), B^{*}}(x)+k\right] d x=-K .
\end{aligned}
$$


Proof. By Lemma 5.8 and the inequality in (5.47), for $B \in\left(\underline{B}_{2}, \bar{B}\right)$, we have $A^{*}(B) \in(\underline{A}(B), \bar{A}(B))$. From (5.36), it follows that

$$
g_{A^{*}(B), B}\left(x_{1}(B)\right)=A^{*}(B)-h\left(x_{1}(B)\right) / \mu<-k .
$$

Therefore, there exist unique $d(B)$ and $D(B)$ such that

$$
\begin{aligned}
& d(B)<x_{1}(B)<D(B)<U\left(A^{*}(B), B\right), \\
& g_{A^{*}(B), B}(d(B))=g_{A^{*}(B), B}(D(B))=-k, \\
& g_{A^{*}(B), B}^{\prime}(d(B))<0, g_{A^{*}(B), B}^{\prime}(D(B))>0 .
\end{aligned}
$$

Let

$$
\Lambda_{1}\left(A^{*}(B), B\right)=\int_{d(B)}^{D(B)}\left[g_{A^{*}(B), B}(x)+k\right] d x .
$$

We now prove that $\Lambda_{1}\left(A^{*}(B), B\right)$ is continuous and strictly decreasing in $B \in\left(\underline{B}_{2}, \bar{B}\right)$ and

$$
\lim _{B \downarrow \underline{B}_{2}} \Lambda_{1}\left(A^{*}(B), B\right)=0 \quad \text { and } \quad \lim _{B \uparrow \bar{B}} \Lambda_{1}\left(A^{*}(B), B\right)=-\infty .
$$

Therefore, there exists a unique $B^{*} \in\left(\underline{B}_{2}, \bar{B}\right)$ such that

$$
\Lambda_{1}\left(A^{*}\left(B^{*}\right), B^{*}\right)=-K,
$$

from which we prove the lemma.

To prove that $\Lambda_{1}\left(A^{*}(B), B\right)$ is continuous and strictly decreasing in $B \in$ $\left(\underline{B}_{2}, \bar{B}\right)$, we apply the Implicit Function Theorem to (5.53). We have

$$
\frac{d A^{*}(B)}{d B}=\frac{\int_{U\left(A^{*}(B), B\right)}^{u\left(A^{*}(B)\right.} e^{-\lambda(x-a)} d x}{u\left(A^{*}(B), B\right)-U\left(A^{*}(B), B\right)}>0 .
$$

Equation (5.56) yields that, for $x \in[d(B), D(B)]$

$$
\begin{aligned}
\frac{\partial g_{A^{*}(B), B}(x)}{\partial B} & =\frac{d A^{*}(B)}{d B}-e^{-\lambda(x-a)} \\
& =\frac{\int_{U\left(A^{*}(B), B\right)}^{u(B)}\left[e^{-\lambda(y-a)}-e^{-\lambda(x-a)}\right] d y}{u\left(A^{*}(B), B\right)-U\left(A^{*}(B), B\right)} \\
& <0 .
\end{aligned}
$$

This in turn implies

$$
\frac{\partial \Lambda_{1}\left(A^{*}(B), B\right)}{\partial B}=\int_{d(B)}^{D(B)} \frac{\partial g_{A^{*}(B), B}(x)}{\partial B} d x<0 .
$$

Therefore, $\Lambda_{1}\left(A^{*}(B), B\right)$ is strictly decreasing in $B \in\left(\underline{B}_{2}, \bar{B}\right)$. 
From (5.47) and Lemma 5.8, it follows that

$$
A^{*}\left(\underline{B}_{2}\right)=\bar{A}\left(\underline{B}_{2}\right) .
$$

This, together with the definition of $\bar{A}(B)$ in (5.36), shows

$$
g_{A^{*}\left(\underline{B}_{2}\right), \underline{B}_{2}}\left(x_{1}\left(\underline{B}_{2}\right)\right)=g_{\bar{A}\left(\underline{B}_{2}\right), \underline{B}_{2}}\left(x_{1}\left(\underline{B}_{2}\right)\right)=\bar{A}\left(\underline{B}_{2}\right)-\frac{1}{\mu} h\left(x_{1}\left(\underline{B}_{2}\right)\right)=-k .
$$

Therefore,

$$
\lim _{B \downarrow \underline{B}_{2}} D(B)=\lim _{B \downarrow \underline{B}_{2}} d(B)=x_{1}\left(\underline{B}_{2}\right) .
$$

It follows that

$$
\lim _{B \downarrow \underline{B}_{2}} \Lambda_{1}\left(A^{*}(B), B\right)=0 .
$$

It remains to prove

$$
\lim _{B \uparrow \bar{B}} \Lambda_{1}\left(A^{*}(B), B\right)=-\infty
$$

For $B \in\left(\underline{B}_{2}, \bar{B}\right)$,

$$
\begin{aligned}
\frac{\partial g_{A^{*}(B), B}\left(x_{1}(B)\right)}{\partial B}=\frac{d A^{*}(B)}{d B} & \\
& -e^{-\lambda\left(x_{1}(B)-a\right)}+g_{A^{*}(B), B}^{\prime}\left(x_{1}(B)\right) \frac{d x_{1}(B)}{d B} \\
= & \frac{\int_{U\left(A^{*}(B), B\right)}^{u\left(A^{*}(B), B\right)}\left[e^{-\lambda(y-a)}-e^{-\lambda\left(x_{1}(B)-a\right)}\right] d y}{u\left(A^{*}(B), B\right)-U\left(A^{*}(B), B\right)} \\
< & 0 .
\end{aligned}
$$

Fix a $\underline{B}_{3} \in\left(\underline{B}_{2}, \bar{B}\right)$ and let

$$
M_{1}=\left(-k-g_{A^{*}\left(\underline{B}_{3}\right), \underline{B}_{3}}\left(x_{1}\left(\underline{B}_{3}\right)\right)\right) / 2 .
$$

From (5.54), it follows that $M_{1}>0$. Then (5.60) implies that for each $B \in\left(\underline{B}_{3}, \bar{B}\right)$,

$$
g_{A^{*}(B), B}\left(x_{1}(B)\right)<g_{A^{*}\left(\underline{B}_{3}\right), \underline{B}_{3}}\left(x_{1}\left(\underline{B}_{3}\right)\right)=-k-2 M_{1}<-k-M_{1} .
$$

Therefore, for each $B \in\left(\underline{B}_{3}, \bar{B}\right)$ there exist unique $d_{1}(B)$ and $D_{1}(B)$ such that

$$
\begin{aligned}
& d_{1}(B)<x_{1}(B)<D_{1}(B), \\
& g_{A^{*}(B), B}\left(d_{1}(B)\right)=g_{A^{*}(B), B}\left(D_{1}(B)\right)=-k-M_{1}, \\
& g_{A^{*}(B), B}^{\prime}\left(d_{1}(B)\right)<0, g_{A^{*}(B), B}^{\prime}\left(D_{1}(B)\right)>0 .
\end{aligned}
$$


The properties of $g$ in Lemma 5.2 (see also Figure 1) imply that for each $B \in\left(\underline{B}_{3}, \bar{B}\right)$

$$
d(B)<d_{1}(B)<x_{1}(B)<D_{1}(B)<D(B) .
$$

This, together with (5.31), implies

$$
\lim _{B \uparrow \bar{B}} d_{1}(B)=-\infty
$$

Note that for $x \in(d(B), D(B)), g_{A^{*}(B), B}(x)<-k$. Therefore, for $B \in$ $\left(\underline{B}_{3}, \bar{B}\right)$,

$$
\begin{aligned}
\Lambda_{1}\left(A^{*}(B), B\right) & =\int_{d(B)}^{D(B)}\left[g_{A^{*}(B), B}(x)+k\right] d x \\
& \leq \int_{d_{1}(B)}^{D_{1}(B)}\left[g_{A^{*}(B), B}(x)+k\right] d x \\
& \leq \int_{d_{1}(B)}^{D_{1}(B)}\left[-M_{1}\right] d x \\
& =-M_{1}\left(D_{1}(B)-d_{1}(B)\right) .
\end{aligned}
$$

From (5.61) and (5.56), it follows that for each $B \in\left(\underline{B}_{3}, \bar{B}\right)$,

$$
\frac{d D_{1}(B)}{d B}=\frac{\int_{U\left(A^{*}(B), B\right)}^{u\left(A^{*}(B), B\right)}\left[e^{-\lambda\left(D_{1}(B)-a\right)}-e^{-\lambda(x-a)}\right] d x}{\left(u\left(A^{*}(B), B\right)-U\left(A^{*}(B), B\right)\right) g^{\prime}\left(D_{1}(B)\right)}>0 .
$$

Thus, for any $B \in\left(\underline{B}_{3}, \bar{B}\right)$,

$$
D_{1}(B) \geq D_{1}\left(\underline{B}_{3}\right) .
$$

Thus, for any $B \in\left(\underline{B}_{3}, \bar{B}\right)$,

$$
\Lambda_{1}\left(A^{*}(B), B\right) \leq M_{1} d_{1}(B)-M_{1} D_{1}\left(\underline{B}_{3}\right) .
$$

Now (5.59) readily follows from (5.63) and (5.62).

6. Singular controls. Assume that $K=0$ and $L=0$. Our feasible policies $\left(Y_{1}, Y_{2}\right)$ in $(2.1)$ are all adaptive, nondecreasing processes that include singular controls, also known as instantaneous controls. Under a singular control, $\left(Y_{1}, Y_{2}\right)$ has infinitely many increases in each finite interval $[0, t]$. An example of a singular control policy is a two-parameter control band policy, which is defined by two parameters $d$, $u$ with $d<u$. No control is exercised until the inventory level $Z(t)$ reaches the lower boundary $d$ or the upper boundary $u$. When $Z(t)$ reaches a boundary, there is no advantage in using impulse control because there is no fixed cost. 
6.1. Control band policies. Let us fix a two-parameter control band policy $\varphi=\{d, u\}$. To mathematically describe the control process $\left(Y_{1}, Y_{2}\right)$, we need to use a two-sided regulator: for each $x \in \mathbb{D}$ with $x(0) \in[d, u]$, find a triple $\left(y_{1}, y_{2}, z\right) \in \mathbb{D}^{3}$ such that

$$
\begin{aligned}
& z(t)=x(t)+y_{1}(t)-y_{2}(t), \quad t \geq 0 \\
& z(t) \in[d, u], \quad t \geq 0, \\
& y_{1}(0)=y_{2}(0)=0, \quad y_{1} \text { and } y_{2} \text { are nondecreasing, } \\
& y_{1} \text { and } y_{2} \text { increases only when } z=d \text { and } z=u, \text { respectively. }
\end{aligned}
$$

The precise mathematical meaning of (6.4) is

$$
\int_{0}^{\infty}(z(t)-d) d y_{1}(t)=0 \quad \text { and } \quad \int_{0}^{\infty}(u-z(t)) d y_{2}(t)=0 .
$$

We can verify that (6.5) is equivalent to the following: whenever $z(t)>d$ for $t \in\left[t_{1}, t_{2}\right], y_{1}\left(t_{2}\right)-y_{1}\left(t_{1}\right)=0$ and whenever $z(t)<u$ for $t \in\left[t_{1}, t_{2}\right]$, $y_{2}\left(t_{2}\right)-y_{2}\left(t_{1}\right)=0$. Lemma 6.1 below follows from Proposition 6 in Section 2.4 of [40]. That proposition is stated for each continuous path $x \in \mathbb{D}$; we can verify that the proposition continues to hold when the continuity of $x$ is dropped.

Lemma 6.1. For each $x \in \mathbb{D}$ with $x(0) \in[d, u]$, there exists a unique triple $\left(y_{1}, y_{2}, z\right) \in \mathbb{D}^{3}$ that satisfies (6.1)-(6.5).

The lemma asserts that the map $\Psi: x \in \mathbb{D}_{0} \rightarrow\left(y_{1}, y_{2}, z\right) \in \mathbb{D}^{3}$ is well defined, where $\mathbb{D}_{0}=\{x \in \mathbb{D}: x(0) \in[d, u]\}$. In the following, we use notation

$$
y_{1}=\Psi_{1}(x), \quad y_{2}=\Psi_{2}(x), \quad \text { and } z=\Psi_{3}(x) .
$$

The nondecreasing functions $\left(y_{1}, y_{2}\right)$ are said to be the two-sided regulator of $x$, and $z$ is the regulated path of $x$. When either $u=\infty$ or $d=-\infty$, the corresponding one-sided regular is defined in Section 2.2 of [40].

Under control band policy $\{d, u\}$ with initial inventory level $x \in[d, u]$, controls $\left(Y_{1}, Y_{2}\right)$ are given by $Y_{1}=\Psi_{1}(X), Y_{2}=\Psi_{2}(X)$, and inventory process $Z=\Psi_{3}(X)$.

To find the long-run average cost under the policy $\varphi=\{d, u\}$, we use the following theorem.

TheOrem 6.1. Fix a control band policy $\varphi=\{d, u\}$. If there exists a constant $\gamma$ and a twice continuously differentiable function $V:[d, u] \rightarrow \mathbb{R}$ that satisfies

$$
\Gamma V(x)+h(x)=\gamma, \quad d \leq x \leq u,
$$


with boundary conditions

$$
\begin{aligned}
& V^{\prime}(d)=-k, \\
& V^{\prime}(u)=\ell,
\end{aligned}
$$

then the average cost $A C(x, \varphi)$ is independent of the initial inventory level $x \in \mathbb{R}$ and is given by $\gamma$ in (6.6).

Proof. First, we assume $x \in[d, u]$. In this case, $Z(0)=x$. By Itô's formula,

$$
\begin{aligned}
V(Z(t))= & V(Z(0))+\int_{0}^{t} \Gamma V(Z(s)) d s+\sigma \int_{0}^{t} V^{\prime}(Z(s)) d W(s) \\
& +\int_{0}^{t} V^{\prime}(Z(s)) d Y_{1}(s)-\int_{0}^{t} V^{\prime}(Z(s)) d Y_{2}(s) \\
= & V(Z(0))+\int_{0}^{t} \Gamma V(Z(s)) d s+\sigma \int_{0}^{t} V^{\prime}(Z(s)) d W(s) \\
& +V^{\prime}(d) Y_{1}(t)-V^{\prime}(u) Y_{2}(t) \\
= & V(Z(0))+\gamma t-\int_{0}^{t} h(Z(s)) d s+\sigma \int_{0}^{t} V^{\prime}(Z(s)) d W(s) \\
& -k Y_{1}(t)-\ell Y_{2}(t) .
\end{aligned}
$$

Therefore

$$
\mathbb{E}_{x}[V(Z(t))]=\mathbb{E}_{x}[V(Z(0))]+\gamma t-\mathbb{E}_{x}\left(\int_{0}^{t} h(Z(s)) d s+k Y_{1}(t)+\ell Y_{2}(t)\right) .
$$

Dividing both sides by $t$ and taking the limit as $t \rightarrow \infty$, we have $\operatorname{AC}(x, \varphi)=\gamma$.

When $x \notin[d, u]$, we assume $Z$ immediately jumps to the closest point in $[d, u]$ at time 0 . Therefore, $Z(0)=d$ if $x<d$ and $Z(0)=u$ if $x>u$. Since $Z(0) \in[d, u]$, the remainder of the proof is identical to the case when $x \in[d, u]$.

Proposition 2. Let $\varphi=\{d, u\}$ be a control band policy with

$$
d<u \text {. }
$$

Let $m \in \mathbb{R}$ be any fixed number. Define

$$
V(x)=\int_{m}^{x} g(y) d y
$$


with

$$
g(x)=V^{\prime}(m) e^{\lambda(m-x)}+\gamma \frac{2}{\sigma^{2}} \int_{m}^{x} e^{\lambda(y-x)} d y-\frac{2}{\sigma^{2}} \int_{m}^{x} h(y) e^{\lambda(y-x)} d y,
$$

where

$$
\begin{gathered}
\gamma=\frac{d_{1}\left(f_{2}+\ell\right)+d_{2}\left(f_{1}+k\right)}{d_{1} e_{2}+d_{2} e_{1}}, \\
V^{\prime}(m)=\frac{e_{1}\left(f_{2}+\ell\right)+e_{2}\left(f_{1}+k\right)}{d_{1} e_{2}+d_{2} e_{1}} .
\end{gathered}
$$

Then $(V, \gamma)$ is a solution to (6.6)-(6.8). In (6.9) and (6.10), set

$$
\begin{aligned}
& d_{1}=e^{\lambda(m-d)}, \quad d_{2}=e^{\lambda(m-u)}, \\
& e_{1}=-\frac{2}{\sigma^{2}} \int_{m}^{d} e^{\lambda(y-d)} d y, \quad e_{2}=\frac{2}{\sigma^{2}} \int_{m}^{u} e^{\lambda(y-u)} d y, \\
& f_{1}=-\frac{2}{\sigma^{2}} \int_{m}^{d} h(y) e^{\lambda(y-d)} d y, \quad f_{2}=\frac{2}{\sigma^{2}} \int_{m}^{u} h(y) e^{\lambda(y-u)} d y .
\end{aligned}
$$

Proof. Similar to the proof of Proposition 1, equation (6.6) implies

$$
V^{\prime}(x)=e^{\lambda(m-x)} V^{\prime}(m)+\gamma \frac{2}{\sigma^{2}} \int_{m}^{x} e^{\lambda(y-x)} d y-\frac{2}{\sigma^{2}} \int_{m}^{x} h(y) e^{\lambda(y-x)} d y .
$$

Boundary conditions (6.7) and (6.8) become

$$
\begin{array}{r}
e^{\lambda(m-d)} V^{\prime}(m)+\gamma \frac{2}{\sigma^{2}} \int_{m}^{d} e^{\lambda(y-d)} d y \\
-\frac{2}{\sigma^{2}} \int_{m}^{d} h(y) e^{\lambda(y-d)} d y=-k, \\
e^{\lambda(m-u)} V^{\prime}(m)+\gamma \frac{2}{\sigma^{2}} \int_{m}^{u} e^{\lambda(y-u)} d y \\
-\frac{2}{\sigma^{2}} \int_{m}^{u} h(y) e^{\lambda(y-u)} d y=\ell .
\end{array}
$$

Using the coefficients defined in (6.11)-(6.13), we see the boundary conditions (6.14) and (6.15) become

$$
\begin{aligned}
& d_{1} V^{\prime}(m)-\gamma e_{1}=-\left(k+f_{1}\right), \\
& d_{2} V^{\prime}(m)+\gamma e_{2}=\ell+f_{2},
\end{aligned}
$$

from which we have the unique solution for $\gamma$ and $V^{\prime}(m)$ given in (6.9) and (6.10). 
6.2. Optimal policy and optimal parameters. Theorem 4.1 suggests the following strategy to obtain an optimal policy. We hope the optimal policy is a control band policy. Therefore, the first task is to find an optimal control band policy among all control band policies. Denote this optimal control band policy by $\varphi^{*}=\left\{d^{*}, u^{*}\right\}, d^{*}<u^{*}$, with long-run average cost $\gamma^{*}$. We hope that $\gamma^{*}$ can be used as the constant in (4.1) of Theorem 4.1. To find the corresponding $f$ that satisfies all of the conditions of Theorem 4.1, we start with the relative value function $V(x)$ associated with the policy $\varphi^{*}$ that is defined on the interval $\left[d^{*}, u^{*}\right]$. We need to extend $V(x)$ so that it can be defined on $\mathbb{R}$. Given that $V(x)$ is the relative value function, it is natural to define

$$
f(x)= \begin{cases}V\left(d^{*}\right)+k\left(d^{*}-x\right) & \text { for } x<d^{*} \\ V(x) & \text { for } d^{*} \leq x \leq u^{*} \\ V\left(u^{*}\right)+\ell\left(x-u^{*}\right) & \text { for } x>u^{*}\end{cases}
$$

Since we wish $f \in C^{1}(\mathbb{R})$, we should have

$$
V^{\prime}\left(d^{*}\right)=-k, \quad V^{\prime}\left(u^{*}\right)=\ell .
$$

Since we also wish $f \in C^{2}(\mathbb{R})$, we should also have the following conditions,

$$
V^{\prime \prime}\left(d^{*}+\right)=0, \quad V^{\prime \prime}\left(u^{*}-\right)=0 .
$$

To explain why condition $V^{\prime \prime}\left(d^{*}+\right)=0$ is needed, we follow Harrison and Taksar [44, p. 451]. Because $K=0$, condition (4.2) is equivalent to $f^{\prime}(x) \geq$ $-k$ for all $x \in \mathbb{R}$. If $V^{\prime \prime}\left(d^{*}+\right)<0,(6.17)$ implies that there exists an $\epsilon>0$ such that $V^{\prime}\left(d^{*}+\epsilon\right)<-k$, which violates lower bound condition $f^{\prime}\left(d^{*}+\right.$ $\epsilon)=V^{\prime}\left(d^{*}+\epsilon\right) \geq-k$. On the other hand, suppose $V^{\prime \prime}\left(d^{*}+\right)>0$. Since $\Gamma V\left(d^{*}+\right)+h\left(d^{*}+\right)=\gamma, f^{\prime}$ and $h$ are continuous in $\mathbb{R}, f^{\prime \prime}\left(d^{*}-\right)=0$, we have $\Gamma f\left(d^{*}-\right)+h\left(d^{*}-\right)<\gamma$, which violates lower bound condition (4.1) for each $x<d^{*}$. Thus, it must be true that $V^{\prime \prime}\left(d^{*}+\right)=0$. Condition $V^{\prime \prime}\left(u^{*}-\right)=0$ can be explained similarly.

In this section, we first prove the existence of parameters $d^{*}$ and $u^{*}$ such that the relative value function $V$ corresponding to control band policy $\varphi=\left\{d^{*}, u^{*}\right\}$ satisfies (6.6)-(6.8), and (6.17)-(6.18). Since part of the solution is to find the boundary points $d^{*}$ and $u^{*},(6.6)-(6.8)$ and (6.17)-(6.18) define a free boundary problem. We then prove that the extension $f$ in (6.16) and $\gamma^{*}=\mathrm{AC}\left(\varphi^{*}, x\right)$ jointly satisfy all of the conditions in Theorem 4.1.

In the remainder of this section, we assume that $\mu>0$. The statement and analysis for the cases $\mu<0$ and $\mu=0$ are analogous and are omitted. Recall the function $g(x)=g_{A, B}(x)$ defined in (5.20). 


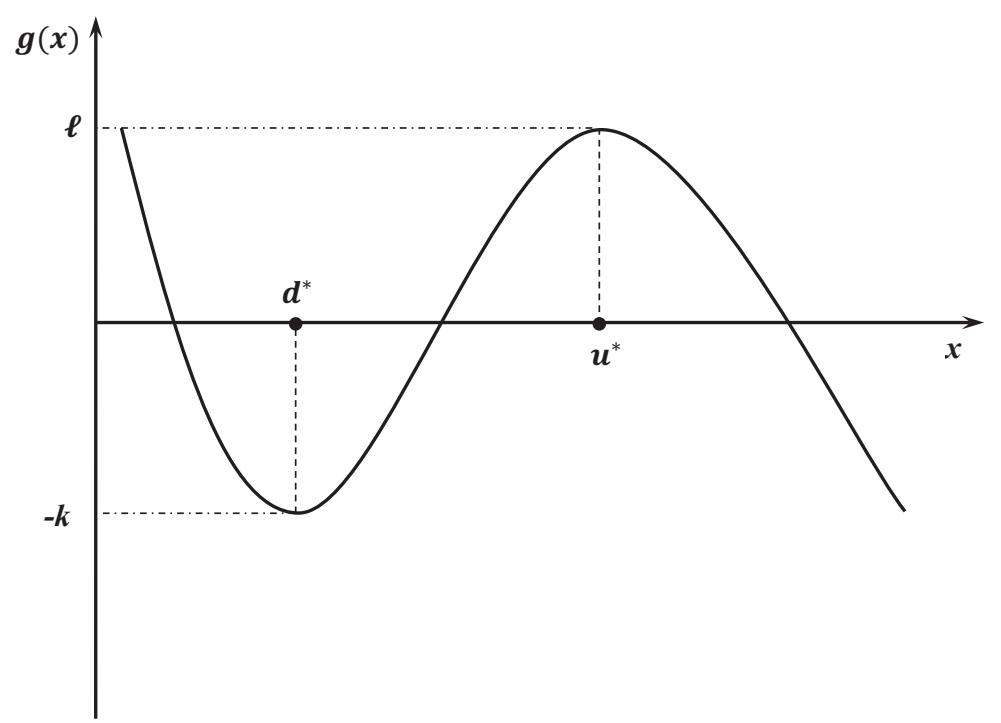

FIG 2. There exist unique $d^{*}=x_{1}\left(\underline{B}_{1}\right)$ and $u^{*}=x_{2}\left(\underline{B}_{1}\right)$.

Theorem 6.2. Assume that h satisfies Assumption 1. There exist unique $A^{*}, B^{*}, d^{*}$ and $u^{*}$ such that $g(x)=g_{A^{*}, B^{*}}(x), d^{*}$ and $u^{*}$ satisfy

$$
\begin{aligned}
& g\left(d^{*}\right)=-k, \\
& g\left(u^{*}\right)=\ell, \\
& g^{\prime}\left(d^{*}\right)=0, \\
& g^{\prime}\left(u^{*}\right)=0 .
\end{aligned}
$$

Furthermore, $g(x)$ decreases in $\left(-\infty, d^{*}\right)$, increases in $\left(d^{*}, u^{*}\right)$, and decreases again in $\left(u^{*}, \infty\right)$.

Proof. Recall the definition of $\bar{B}$ in (5.25). For each $B \in(0, \bar{B})$, by Lemma 5.2, there is a unique local minimizer $x_{1}(B)<a$ and a unique local maximizer $x_{2}(B)>a$ for function $g_{A, B}(x)$. By Lemma 5.4, there exists a unique $\underline{B}_{1} \in(0, \bar{B})$ that satisfies $(5.39)$. Let

$$
A^{*}=h\left(x_{1}\left(\underline{B}_{1}\right)\right) / \mu-k=h\left(x_{2}\left(\underline{B}_{1}\right)\right) / \mu+\ell
$$

and $B^{*}=\underline{B}_{1}$. Then $g(x)=g_{A^{*}, \underline{B}_{1}}(x), d^{*}=x_{1}\left(\underline{B}_{1}\right)$ and $u^{*}=x_{2}\left(\underline{B}_{1}\right)$ satisfy (6.19)-(6.22); see Figure 2.

Now we show that control band policy $\varphi^{*}=\left\{d^{*}, u^{*}\right\}$ is the optimal policy among all feasible policies. 
Theorem 6.3. Assume that $h$ satisfies Assumption 1. Let $d^{*}$ and $u^{*}$, along with constants $A^{*}$ and $B^{*}$, be the unique solution in Theorem 6.2. Then control band policy $\varphi^{*}=\left\{d^{*}, u^{*}\right\}$ is optimal among all feasible policies.

Proof. Let $g(x), x \in \mathbb{R}$, be the function in (5.20) with $A=A^{*}$ and $B=B^{*}$. Let

$$
\bar{g}(x)= \begin{cases}-k & \text { for } x<d^{*} \\ g(x) & \text { for } d^{*} \leq x \leq u^{*} \\ \ell & \text { for } x>u^{*}\end{cases}
$$

Define

$$
V(x)=\int_{d^{*}}^{x} \bar{g}(y) d y
$$

Let $\gamma^{*}$ be the long-run average cost under policy $\varphi^{*}$. We now show that $V$ and $\gamma^{*}$ satisfy all of the conditions in Theorem 4.1. Thus, Theorem 4.1 shows that the long-run average cost under any policy is at least $\gamma^{*}$. Therefore, $\gamma^{*}$ is the optimal cost and control band policy $\varphi^{*}$ is an optimal policy. Now we check that $V(x)$ is in $C^{2}(\mathbb{R})$ and satisfies (4.1)-(4.3).

First, $V(x)$ is in $C^{2}\left(\left[d^{*}, u^{*}\right]\right)$. Theorem 6.2 and the definition of $V$ in (6.23) imply

$$
\lim _{x \uparrow d^{*}} V^{\prime \prime}(x)=0=\lim _{x \downarrow d^{*}} V^{\prime \prime}(x), \text { and } \lim _{x \uparrow u^{*}} V^{\prime \prime}(x)=0=\lim _{x \downarrow u^{*}} V^{\prime \prime}(x) .
$$

Then, $V^{\prime \prime}(x)$ is continuous at $d^{*}$ and $u^{*}$. Note that $V^{\prime \prime}(x)=0$ in $\left(-\infty, d^{*}\right)$ and $\left(u^{*},+\infty\right)$. Therefore, $V(x)$ is in $C^{2}(\mathbb{R})$. Let

$$
M=\sup _{x \in\left[d^{*}, u^{*}\right]}|g(x)|,
$$

we have $\left|V^{\prime}(x)\right| \leq M$ for all $x \in \mathbb{R}$.

To check (4.1), we first find that $\Gamma V(x)+h(x)=\gamma^{*}$ for $d^{*} \leq x \leq u^{*}$. For $x<d^{*}$,

$$
\begin{aligned}
\Gamma V(x)+h(x) & =\frac{\sigma^{2}}{2} V^{\prime \prime}(x)+\mu V^{\prime}(x)+h(x) \\
& =\frac{\sigma^{2}}{2} V^{\prime \prime}\left(d^{*}\right)+\mu V^{\prime}\left(d^{*}\right)+h(x) \\
& \geq \frac{\sigma^{2}}{2} V^{\prime \prime}\left(d^{*}\right)+\mu V^{\prime}\left(d^{*}\right)+h\left(d^{*}\right) \\
& =\gamma^{*},
\end{aligned}
$$


where the second equality is due to $V^{\prime \prime}(x)=0=V^{\prime \prime}\left(d^{*}\right)$ and $V^{\prime}(x)=-k=$ $V^{\prime}\left(d^{*}\right)$ for $x<d^{*}$, the inequality is due to $x<d^{*}=x_{1}<a$, where $a$ again is the minimum point of $h$. Similarly, for $x>u^{*}, \Gamma V(x)+h(x) \geq \gamma^{*}$.

Finally, (4.2) and (4.3) hold because $\bar{g}(x)$ is strictly increasing in $x, x \in$ $\left[d^{*}, u^{*}\right]$, and $\bar{g}\left(d^{*}\right)=g\left(d^{*}\right)=-k, \bar{g}\left(u^{*}\right)=g\left(u^{*}\right)=\ell$; see Figure 2. Thus, the optimality of control band policy $\varphi^{*}$ is implied by Theorem 4.1 .

7. No inventory backlog. In this section, the inventory backlog is not allowed and thus we add the constraint $Z(t) \geq 0$ for all $t \geq 0$. Holding cost function $h(\cdot)$ is defined on $[0, \infty)$, and $a \in[0, \infty)$ is its minimum point. We focus on the impulse control case when $K>0$ and $L>0$. The results and proofs in this section are analogous to those discussed in Section 5. Here, we highlight the differences. Other cases when $K=0$ and $L=0$, or when $K>0$ and $L=0$, or when $K=0$ and $L>0$ can be studied analogously. For example, the optimal policy for the case when $K=0$ and $L=0$ with constraint $Z(t) \geq 0$ can be obtained by extending Section 6 to the non-backlog setting.

For control band policy $\{d, D, U, u\}$ with $0 \leq d<D<U<u$, we continue to use Theorem 5.1 to evaluate its performance and to obtain its relative value function. However, we need to modify Theorem 4.1, the lower bound theorem, slightly as follows.

Theorem 7.1. Suppose that $f \in C^{1}([0,+\infty))$ and $f^{\prime}$ is absolutely continuous such that $f^{\prime \prime}$ is locally $L^{1}$. Suppose there exists a constant $M>0$ such that $\left|f^{\prime}(x)\right| \leq M$ for all $x \in[0,+\infty)$. Assume further

$$
\begin{aligned}
& \Gamma f(x)+h(x) \geq \gamma \quad \text { for } x \in[0,+\infty), \\
& f(y)-f(x) \leq K+k(x-y) \quad \text { for } 0 \leq y<x, \\
& f(y)-f(x) \leq L+\ell(y-x) \quad \text { for } 0 \leq x<y .
\end{aligned}
$$

Then $A C(x, \varphi) \geq \gamma$ for each feasible policy $\varphi$ and each initial state $x \in$ $[0,+\infty)$.

7.1. Optimal policy parameters. Recall that for control band policy $\{d, D$, $U, u\}$ with $0 \leq d<D<U<u$, the corresponding relative value function satisfies (5.1)-(5.3). To search for the optimal parameters $\left(d^{*}, D^{*}, U^{*}, u^{*}\right)$, we impose the following conditions on $\{d, D, U, u\}$ and $V$ :

$$
\begin{aligned}
& V^{\prime}(U)=l, \\
& V^{\prime}(u)=l, \\
& V^{\prime}(D)=-k,
\end{aligned}
$$




$$
\begin{aligned}
& V^{\prime}(d)=-k-\alpha, \\
& 0 \leq d<D<U<u \\
& \alpha d=0, \text { and } \\
& \alpha \geq 0 .
\end{aligned}
$$

In some cases, it is optimal to have $d^{*}=0$. Then, we need only to solve for three parameters $D^{*}, U^{*}$ and $u^{*}$. This section is analogous to Section 5.2. Here, we highlight the differences, omitting some details to avoid repetition.

Recall that $a$ is the minimum point of holding cost function $h(x)$ on $[0, \infty)$. It is possible $a=0$ or $a>0$. In the following, whenever we invoke Assumption 1 for $h$, we ignore any condition on $h(x)$ with $x<0$. Similar to Lemma 5.1, we have the following lemma.

Lemma 7.1. For each $A, B \in \mathbb{R}$, function $g(x)=g_{A, B}(x)$ in (5.20) is a solution to equation

$$
\Gamma g(x)+h^{\prime}(x)=0 \quad \text { for all } x \in[0, \infty) \backslash\{a\} .
$$

The following theorem solves the free boundary problem when inventory backlog is not allowed.

THEOREM 7.2. Assume that holding cost function $h$ satisfies conditions (a)-(d) of Assumption 1. There exist unique $A^{*}, B^{*}, d^{*}, D^{*}, U^{*}, u^{*}, x_{1}^{*}, x_{2}^{*}$ and $\alpha^{*}$ with

$$
0 \leq d^{*} \leq x_{1}^{*}<D^{*} \quad \text { and } \quad U^{*}<x_{2}^{*}<u^{*}
$$

such that the corresponding $g(x)=g_{A^{*}, B^{*}}(x)$ satisfies

$$
\begin{aligned}
& \int_{d^{*}}^{D^{*}}[g(x)+k] d x=-K, \\
& \int_{U^{*}}^{d^{*}}[g(x)-\ell] d x=L, \\
& g\left(d^{*}\right)=-k-\alpha^{*}, \quad g\left(D^{*}\right)=-k, \\
& g\left(U^{*}\right)=g\left(u^{*}\right)=\ell, \\
& \alpha^{*} d^{*}=0, \quad \text { and } \\
& \alpha^{*} \geq 0 .
\end{aligned}
$$

Furthermore, $g$ has a local minimum in $[0, a]$ at $x_{1}^{*} \in[0, a]$ and $g$ has the maximum at $x_{2}^{*} \in(a, \infty)$. Function $g$ is decreasing in $\left(0, x_{1}^{*}\right)$, increasing in $\left(x_{1}^{*}, x_{2}^{*}\right)$ and decreasing again in $\left(x_{2}^{*}, \infty\right)$.

We discuss the proof of Theorem 7.2 at the end of this section. 
THEOREM 7.3. Assume that holding cost function $h$ satisfies conditions (a)-(d) of Assumption 1. Let $0 \leq d^{*}<D^{*}<U^{*}<u^{*}$, along with constants $A^{*}$ and $B^{*}$, be the unique solution in Theorem 7.2. Then control band policy $\varphi^{*}=\left\{d^{*}, D^{*}, U^{*}, u^{*}\right\}$ is optimal among all feasible policies to minimize the long-run average cost when inventory backlog is not allowed.

Proof. The proof is identical to that of Theorem 5.3.

Now, we discuss the proof for Theorem 7.2. This proof is similar to the proof of Theorem 5.2. We provide an outline of the proof for Theorem 7.2, highlighting the differences between the two proofs. We only consider the case when $\mu>0$. Other cases are analogous and are omitted. Define

$$
\bar{B}_{1}=-\frac{1}{\mu} \int_{0}^{a} h^{\prime}(y) e^{\lambda(y-a)} d y
$$

Because $h^{\prime}(x)<0$ for $x \in(0, a), \bar{B}_{1}>0$.

The following lemma is analogous to Lemma 5.2. The only difference is that the expression for $x_{1}=x_{1}(B)$ has two forms in Lemma 7.2; see Figure 3.

Lemma 7.2. (a) For any $A \in \mathbb{R}$ and for each fixed $B \in(0, \infty), g_{A, B}$ attains a unique minimum in $[0, a]$ at $x_{1}=x_{1}(B) \in[0, a]$. Function $g_{A, B}$ attains a unique maximum in $(a, \infty)$ at $x_{2}=x_{2}(B) \in(a, \infty)$. Both $x_{1}(B)$ and $x_{2}(B)$ are independent of $A$.

(b) For each fixed $B \in(0, \infty)$, the local maximizer $x_{2}=x_{2}(B)$ is the unique solution in $(a, \infty)$ to (5.27). For $B \in\left(0, \bar{B}_{1}\right)$, the local minimizer $x_{1}=x_{1}(B)$ is the unique solution in $(0, a)$ to (5.27). For $B \in\left[\bar{B}_{1}, \infty\right)$, $x_{1}=x_{1}(B)=0$.

(c) For each $B \in(0, \infty), g_{A, B}^{\prime}(x)<0$ for $x \in\left(0, x_{1}(B)\right), g_{A, B}^{\prime}(x)>0$ for $x \in\left(x_{1}(B), x_{2}(B)\right)$, and $g_{A, B}^{\prime}(x)<0$ for $x \in\left(x_{2}(B), \infty\right)$.

The following lemma is analogous to Lemma 5.3.

Lemma 7.3. (a) The local minimizer $x_{1}(B)$ is continuous and nonincreasing in $B \in(0, \infty)$. The local maximizer $x_{2}(B)$ is continuous and strictly increasing in $B \in(0, \infty)$. Furthermore, (5.30) holds and

$$
\lim _{B \uparrow \infty} x_{1}(B)=0, \quad \lim _{B \uparrow \infty} x_{2}(B)=\infty .
$$

(b) For each $B \in(0, \infty)$,

$$
g_{A, B}\left(x_{2}(B)\right)=A-h\left(x_{2}(B)\right) / \mu .
$$




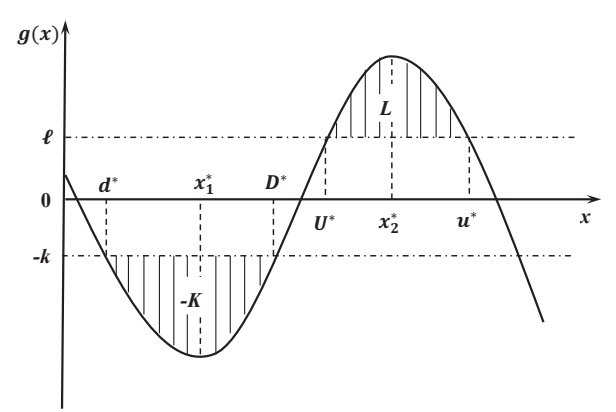

(a) Case $d^{*}>0$ and $x_{1}^{*}>0$

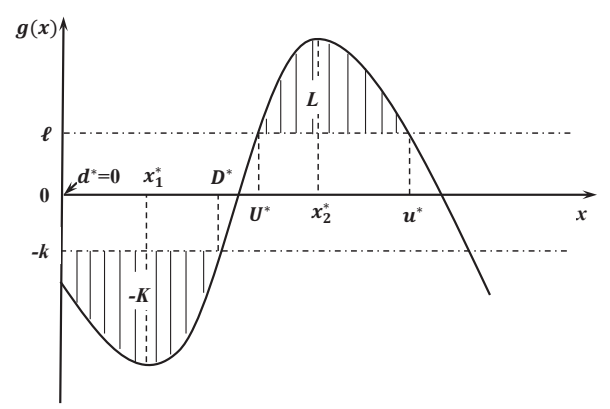

(b) Case $d^{*}=0$ and $x_{1}^{*}>0$

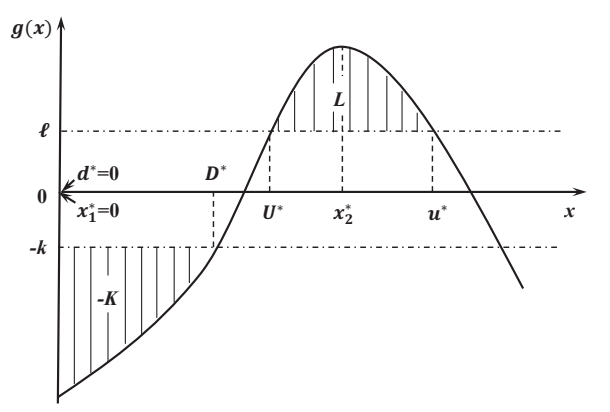

(c) Case $d^{*}=0$ and $x_{1}^{*}=0$

FIG 3. When inventory backlog is not allowed, the parameter $d^{*}$ in the optimal control band policy has two possibilities: $d^{*}>0$ and $d^{*}=0$. When $d^{*}=0$, there are two cases: $g^{\prime}(0)<0$ and $g^{\prime}(0) \geq 0$, corresponding to $x_{1}^{*}>0$ and $x_{1}^{*}=0$, respectively.

For each $B \in\left(0, \bar{B}_{1}\right)$,

$$
g_{A, B}\left(x_{1}(B)\right)=A-h\left(x_{1}(B)\right) / \mu .
$$

For each $B \in\left[\bar{B}_{1}, \infty\right)$,

$$
g_{A, B}\left(x_{1}(B)\right)=g_{A, B}(0)=A-B e^{\lambda a}+\frac{\lambda}{\mu} \int_{0}^{a} h(-y+a) e^{-\lambda(y-a)} d y .
$$

Proof. (a) Note that $x_{1}(B)=0$ for $B \in\left[\bar{B}_{1}, \infty\right)$. Thus, $x_{1}(B)$ is continuous for $B \in\left(\bar{B}_{1}, \infty\right)$. It follows the proof of Lemma 5.3 that $x_{1}(B)$ is continuously differentiable in $B \in\left(0, \bar{B}_{1}\right)$, and $x_{2}(B)$ is continuously differentiable in $B \in(0, \infty)$. We can easily check that $x_{1}(B)$ is continuous at $B=\bar{B}_{1}$ and that $x_{i}(B)$ has the desired monotonicity property for $i=1,2$. The limits in (5.30) can be obtained similarly as in Lemma 5.3. The limit in the left side of (7.8) follows from $x_{1}(B)=0$ for $B \in\left(\bar{B}_{1}, \infty\right)$. The limit in the right side of (7.8) follows from (5.27) for the definition of $x_{2}(B)$. 
(b) Equations (7.9) and (7.10) follow from the proof for (5.32). For $B \in$ $\left[\bar{B}_{1}, \infty\right), x_{1}(B)=0$. Thus, (7.11) follows from (5.20).

Recall the definition $\tilde{g}(B)$ in (5.37) of Lemma 5.4. This time $\tilde{g}(B)$ is well defined for $B \in(0, \infty)$. Recall also the definitions of $\underline{A}(B)$ in $(5.35)$ and $\bar{A}(B)$ in (5.36). We have the following lemma that is analogous to Lemma 5.4.

Lemma 7.4. Function $\tilde{g}(B)$ is independent of $A$. It is continuous and strictly increasing on $B \in(0, \infty)$. Furthermore,

$$
\lim _{B \downarrow 0} \tilde{g}(B)=0 \quad \text { and } \quad \lim _{B \uparrow \infty} \tilde{g}(B)=\infty .
$$

Therefore there exists $\underline{B}_{1} \in(0, \infty)$ such that (5.39) holds. Furthermore, for $B \in\left(\underline{B}_{1}, \infty\right),(5.40)$ holds.

Proof. First, we prove $\tilde{g}$ is strictly increasing. For $B \in\left(0, \bar{B}_{1}\right)$, the expression for $\frac{d \tilde{g}(B)}{d B}$ is identical to the one in $(5.41)$. For $B \in\left(\bar{B}_{1}, \infty\right)$,

$$
\begin{aligned}
\frac{d \tilde{g}(B)}{d B} & =-\frac{1}{\mu} h^{\prime}\left(x_{2}(B)\right) \frac{d x_{2}(B)}{d B}+e^{\lambda a} \\
& =-e^{-\lambda\left(x_{2}(B)-a\right)}+e^{\lambda a} \\
& >0 .
\end{aligned}
$$

Thus, $\tilde{g}$ is strictly increasing.

Next we prove $\lim _{B \uparrow \infty} \tilde{g}(B)=\infty$. We observe that (7.8) and (7.12) imply $\lim _{B \uparrow-\infty} \frac{d \tilde{g}(B)}{d B}=e^{\lambda a}>0$, from which we have $\lim _{B \uparrow \infty} \tilde{g}(B)=\infty$.

The remaining proof of the lemma is identical to that of Lemma 5.4.

With Lemma 7.4 replacing Lemma 5.4, Lemmas 5.5 and 5.6 hold without any modification.

LEMma 7.5. Function $\Lambda_{2}(\bar{A}(B), B)$ is continuous and strictly increasing in $B \in\left(\underline{B}_{1}, \infty\right)$. Furthermore,

$$
\begin{aligned}
& \lim _{B \downarrow \underline{B}_{1}} \Lambda_{2}(\bar{A}(B), B)=0, \\
& \lim _{B \uparrow \infty} \Lambda_{2}(\bar{A}(B), B)=\infty .
\end{aligned}
$$

Therefore, there exists a unique $\underline{B}_{2} \in\left(\underline{B}_{1}, \infty\right)$ such that $(5.47)$ holds.

Proof. The proof of this lemma is identical to the proof of Lemma 5.7 except that we need to prove (7.13). 
To prove (7.13), we follow the expression in (5.48) for $\frac{\partial \Lambda_{2}(\bar{A}(B), B)}{\partial B}$. By (5.51) and (5.52), we know that $U(\bar{A}(B), B)$ decreases in $B$ and $u(\bar{A}(B), B)$ increases in $B$. Also we know that $x_{1}(B)=0$ for $B \in\left(\bar{B}_{1}, \infty\right)$. Following the expression in (5.48) and these facts, we have

$$
\lim _{B \uparrow \infty} \frac{\partial \Lambda_{2}(\bar{A}(B), B)}{\partial B}>0,
$$

which implies (7.13).

Lemma 5.6 and Lemma 7.5 immediately give the following lemma.

Lemma 7.6. For each $B \in\left[\underline{B}_{2}, \infty\right)$, there exists a unique $A^{*}(B) \in$ $(\underline{A}(B), \bar{A}(B)]$ such that (5.53) holds.

Finally, the following lemma gives a proof of Theorem 7.2.

Lemma 7.7. There exist unique $B^{*}$ with $B^{*} \in\left(\underline{B}_{2}, \infty\right), D^{*}, d^{*}, x_{1}^{*}$ and $\alpha^{*}$ with $0 \leq d^{*} \leq x_{1}^{*}<D^{*}$ such that

$$
\begin{aligned}
& g_{A^{*}\left(B^{*}\right), B^{*}}\left(D^{*}\right)=-k, \\
& g_{A^{*}\left(B^{*}\right), B^{*}}\left(d^{*}\right)=-k-\alpha^{*}, \\
& \int_{d^{*}}^{D^{*}}\left[g_{A^{*}\left(B^{*}\right), B^{*}}(x)+k\right] d x=-K, \\
& \alpha^{*} d^{*}=0, \text { and } \\
& \alpha^{*} \geq 0 .
\end{aligned}
$$

Proof. For any $B \in\left(\underline{B}_{2}, \infty\right), A^{*}(B)<\bar{A}(B)$. Therefore, (5.36) implies $g_{A^{*}(B), B}\left(x_{1}(B)\right)<-k$. Thus, there exists a unique $D(B)$ such that

$$
D(B)>0, \quad g_{A^{*}(B), B}(D(B))=-k, \quad g_{A^{*}(B), B}^{\prime}(D(B))>0 .
$$

If $g_{A^{*}(B), B}(0)>-k$, then there exists a unique $d(B)$ such that

$$
d(B) \geq 0, \quad g_{A^{*}(B), B}(d(B))=-k, \quad g_{A^{*}(B), B}^{\prime}(d(B))<0 .
$$

For $B \in\left(\underline{B}_{2}, \infty\right),(5.56)$ implies

$$
\frac{\partial g_{A^{*}(B), B}(0)}{\partial B}=\frac{\int_{U(B)}^{u(B)}\left[e^{-\lambda(x-a)}-e^{\lambda a}\right] d x}{u(B)-U(B)}<0 .
$$

Therefore, $g_{A^{*}(B), B}(0)$ is strictly decreasing in $B \in\left(\underline{B}_{2}, \infty\right)$. Let $\left(\underline{B}_{2}, \underline{B}_{4}\right)$ be the interval over which $g_{A^{*}(B), B}(0)>-k$. If there is no $B$ that satisfies 
$g_{A^{*}(B), B}(0)>-k$, then set $\underline{B}_{4}=\underline{B}_{2}$. Thus, for $B \in\left(\underline{B}_{2}, \bar{B}_{1} \wedge \underline{B}_{4}\right), d(B)>0$. Otherwise, for $B \in\left[\bar{B}_{1} \wedge \underline{B}_{4}, \infty\right)$, set $d(B)=0$. The remainder of the proof mimics the proof of Lemma 5.9.

Define $\Lambda_{1}\left(A^{*}(B), B\right)$ as in (5.55). We will now prove that $\Lambda_{1}\left(A^{*}(B), B\right)$ is continuous and strictly decreasing in $B \in\left[\underline{B}_{2}, \infty\right)$ and

$$
\lim _{B \downarrow \underline{B}_{2}} \Lambda_{1}\left(A^{*}(B), B\right)=0 \quad \text { and } \quad \lim _{B \uparrow \infty} \Lambda_{1}\left(A^{*}(B), B\right)=-\infty .
$$

Therefore, there exists a unique $B^{*} \in\left(\underline{B}_{2}, \infty\right)$, such that

$$
\Lambda_{1}\left(A^{*}\left(B^{*}\right), B^{*}\right)=-K,
$$

from which we prove the lemma by choosing $A^{*}=A^{*}\left(B^{*}\right), D^{*}=D\left(B^{*}\right)$, $d^{*}=d\left(B^{*}\right), x_{1}^{*}=x_{1}^{*}\left(B^{*}\right)$ and $\alpha^{*}=\left(k+g_{A^{*}\left(B^{*}\right), B^{*}}(0)\right)^{-}$.

We first show that $\Lambda_{1}\left(A^{*}(B), B\right)$ is continuous and strictly decreasing in $B \in\left[\underline{B}_{2}, \infty\right)$. Observe that (5.56) continues to hold, from which (5.57) continues to hold. We now claim that (5.57) continues to hold for $B \in$ $\left(\underline{B}_{2}, \infty\right)$ except possibly at $\bar{B}_{1} \wedge \underline{B}_{4}$. Indeed, for $B \in\left(\underline{B}_{2}, \bar{B}_{1} \wedge \underline{B}_{4}\right)$ (5.57) holds as before. For $B \in\left(\bar{B}_{1} \wedge \underline{B}_{4}, \infty\right), d(B)=0$ and (5.57) holds as well in this case. These all prove that $\Lambda_{1}\left(A^{*}(B), B\right)$ is continuous and decreasing in $B$.

Next, it is easy to see that the limit (5.58) continues to hold as well. It remains to prove

$$
\lim _{B \uparrow \infty} \Lambda_{1}\left(A^{*}(B), B\right)=-\infty
$$

We will prove next that

$$
\lim _{B \uparrow \infty} \frac{d \Lambda_{1}\left(A^{*}(B), B\right)}{d B}<0,
$$

from which (7.15) immediately follows.

For (7.16), using (7.14), we have

$$
\begin{aligned}
\frac{d D(B)}{d B} & =\frac{e^{-\lambda(D(B)-a)}-\frac{\int_{U\left(A^{*}(B), B\right)}^{u\left(A^{*}(B), B\right)} e^{-\lambda(x-a)} d x}{u\left(A^{*}(B), B\right)-U\left(A^{*}(B), B\right)}}{g_{A^{*}(B), B}^{\prime}(D(B))} \\
& =\frac{\int_{U\left(A^{*}(B), B\right)}^{u\left(A^{*}(B), B\right)}\left[e^{-\lambda(D(B)-a)}-e^{-\lambda(x-a)}\right] d x}{\left(u\left(A^{*}(B), B\right)-U\left(A^{*}(B), B\right) g_{A^{*}(B), B}^{\prime}(D(B))\right.} \\
& >0 .
\end{aligned}
$$


To study the limit in (7.16), we need only consider $\Lambda_{1}\left(A^{*}(B), B\right)$ for $B \in$ $\left(\bar{B}_{1} \wedge \underline{B}_{4}, \infty\right)$. When $B \in\left(\bar{B}_{1} \wedge \underline{B}_{4}, \infty\right), d(B)=0$ and hence $\Lambda_{1}\left(A^{*}(B), B\right)=$ $\int_{0}^{D(B)}\left[g_{A^{*}(B), B}(x)+k\right] d x$. Therefore, for $B \in\left(\bar{B}_{1} \wedge \underline{B}_{4}, \infty\right)$,

$$
\begin{aligned}
& \frac{d \Lambda_{1}\left(A^{*}(B), B\right)}{d B} \\
& \quad=\int_{0}^{D(B)}\left[\frac{d A^{*}(B)}{d B}-e^{-\lambda(x-a)}\right] d x+\frac{d D(B)}{d B}\left[g_{A^{*}(B), B}(D(B))+k\right] \\
& \quad=\int_{0}^{D(B)}\left[\frac{d A^{*}(B)}{d B}-e^{-\lambda(x-a)}\right] d x,
\end{aligned}
$$

where the last equality is due to $g_{A^{*}(B), B}(D(B))=-k$. From (5.56), it follows that

$$
\begin{aligned}
\frac{d \Lambda_{1}\left(A^{*}(B), B\right)}{d B} & =\int_{0}^{D(B)}\left[\frac{\int_{U(B)}^{u(B)} e^{-\lambda(y-a)} d y}{u(B)-U(B)}-e^{-\lambda(x-a)}\right] d x \\
& \leq \int_{0}^{D(B)}\left[\frac{\int_{U(B)}^{u(B)} e^{-\lambda(D(B)-a)} d y}{u(B)-U(B)}-e^{-\lambda(x-a)}\right] d x \\
& =\int_{0}^{D(B)}\left[e^{-\lambda(D(B)-a)}-e^{-\lambda(x-a)}\right] d x
\end{aligned}
$$

where the inequality follows from $D(B)<U(B)$. Inequality (7.17) implies

$$
\frac{d\left(\int_{0}^{D(B)}\left[e^{-\lambda(D(B)-a)}-e^{-\lambda(x-a)}\right] d x\right)}{d B}=-\lambda e^{-\lambda(D(B)-a)} D(B) \frac{d D(B)}{d B}<0 .
$$

For any $B_{0} \in\left(\bar{B}_{1} \wedge \underline{B}_{4}, \infty\right)$, we have $\int_{0}^{D\left(B_{0}\right)}\left[e^{-\lambda(x-a)}-e^{-\lambda\left(D\left(B_{0}\right)-a\right)}\right] d x<0$. Therefore,

$$
\begin{aligned}
\lim _{B \uparrow \infty} \frac{d \Lambda_{1}\left(A^{*}(B), B\right)}{d B} & \leq \lim _{B \uparrow \infty} \int_{0}^{D(B)}\left[e^{-\lambda(x-a)}-e^{-\lambda(D(B)-a)} d y\right] d x \\
& \leq \int_{0}^{D\left(B_{0}\right)}\left[e^{-\lambda(x-a)}-e^{-\lambda\left(D\left(B_{0}\right)-a\right)}\right] d x \\
& <0,
\end{aligned}
$$

proving (7.16).

Proof of Theorem 7.2. We have defined $B^{*}, d^{*}, D^{*}, x_{1}^{*}$ and $\alpha^{*}$ in Lemma 7.7. As mentioned earlier, Lemma 5.5 continues to hold without any modification in this section. Recall that $U(A, B)$ and $u(A, B)$ are defined 
in Lemma 5.5, $x_{2}(B)$ is defined in Lemma 7.2, and $A^{*}(B)$ is defined in Lemma 7.6. We set $A^{*}=A^{*}\left(B^{*}\right), U^{*}=U\left(A^{*}, B^{*}\right), u^{*}=u\left(A^{*}, B^{*}\right)$ and $x_{2}^{*}=x_{2}\left(B^{*}\right)$. To prove the theorem, it suffices to check that the function $g_{A^{*}, B^{*}}(x)$, together with $A^{*}, B^{*}, d^{*}, D^{*}, U^{*}, u^{*}, x_{1}^{*}, x_{2}^{*}$, and $\alpha^{*}$, satisfies (7.1)(7.7) and the monotonicity properties of $g_{A^{*}, B^{*}}(x)$ as stated in Theorem 7.2. First, Lemma 5.5 implies the second part of (7.1) and (7.5). Second, Lemma 7.6 implies (7.3). Third, Lemma 7.7 implies the first part of (7.1), (7.2), (7.4) and (7.6)-(7.7). Finally, Lemma 7.2 implies the monotonicity properties of $g_{A^{*}, B^{*}}(x)$.

8. Concluding remarks. In this paper, we have given a tutorial of the lower-bound approach for studying the optimal control of Brownian inventory models with a general convex holding cost function. The control can be either impulse or singular, and the inventory can be either backlogged or without backlog. For future research, it would be interesting to study multi-stage inventory systems with Brownian motion demand. Yao [82] has done a preliminary study for these systems.

Acknowledgments. The authors thank the associate editor and two referees for improving the exposition of the paper. They also thank Hanqin Zhang of Chinese Academy of Sciences and National University of Singapore for stimulating discussions. Part of this work was done while the second author was visiting the H. Milton Stewart School of Industrial and Systems Engineering at Georgia Institute of Technology. He thanks the school for its hospitality.

\section{REFERENCES}

[1] Alvarez, L. H. R. (1999) A class of solvable singular stochastic control problems. Stochastics and Stochastic Reports 67 83-122. MR1717803

[2] Alvarez, L. H. R. (2004). Stochastic forest stand value and optimal timber harvesting. SIAM Journal on Control and Optimization 42 1972-1993. MR2080924

[3] Alvarez, L. H. R. (2004). A class of solvable impulse control problems. Applied Mathematics and Optimization 49 265-295. MR2041852

[4] Alvarez, L. H. R. and Koskela, E. (2007). The forest rotation problem with stochastic harvest and amenity value. Natural Resource Modeling 20 477-509. MR2362175

[5] Alvarez, L. H. R. and Koskela, E. (2007). Taxation and rotation age under stochastic forest stand value. Journal of Environmental Economics and Management 54 113-127.

[6] Alvarez, L. H. R. and Lempa, J. (2008). On the optimal stochastic impulse control of linear diffusions. SIAM Journal on Control and Optimization 47 703-732. MR2385860 
[7] Ata, B. (2006). Dynamic control of a multiclass queue with thin arrival streams. Operations Research 54 876-892. MR2267797

[8] AtA, B. and KumAR, S. (2005). Heavy traffic analysis of open processing networks with complete resource pooling: asymptotic optimality of discrete review policies. Annals of Applied Probability 15 331-391. MR2115046

[9] Ata, B. and Olsen, T. L. (2009). Near-optimal dynamic lead-time quotation and scheduling under convex-concave customer delay costs. Operations Research $57753-$ 768 .

[10] BACCARIN, S. (2002). Optimal impulse control for cash management with quadratic holding-penalty costs. Decisions in Economics and Finance 25 19-32. MR1961219

[11] Bar-Ilan, A., Perry, D. and Stadje W. (2004). A generalized impulse control model of cash management. Journal of Economic Dymanics and Control $281013-$ 1033. MR2023596

[12] Bar-Ilan, A. and Sulem, A. (1995). Explicit solution of inventory problems with delivery lags. Mathematics of Operations Research 20 709-720. MR1354778

[13] Bather, J. A.(1966) A continuous time inventory model. Journal of Applied Probability 3 538-549. MR0213148

[14] BAyraktar, E. and EgAmi, M. (2008). An analysis of monotone follower problems for diffusion processes. Mathematics of Operations Research 33 336-350. MR2415997

[15] Bell, S. L. and Williams, R. J. (2005). Dynamic scheduling of a parallel server system in heavy traffic with complete resource pooling: Asymptotic optimality of a threshold policy. Electronic Journal of Probability 10 1044-1115. MR2164040

[16] Bell, S. L. and Williams, R. L. (2001). Dynamic scheduling of a system with two parallel servers in heavy traffic with resource pooling: asymptotic optimality of a threshold policy. Annals of Applied Probability 11 608-649. MR1865018

[17] Benkherouf, L. (2007). On a stochastic inventory model with a generalized holding costs. European Journal of Operational Research 182 730-737.

[18] Benkherouf, L. and Bensoussan, A. (2009). Optimality of an $(s, S)$ policy with compound Poisson and diffusion demands: a quasi-variational inequalities approach. SIAM Journal on Control and Optimization 48 756-762. MR2486092

[19] Bensoussan, A. and Lions, J.-L. (1974/75). Nouvelles méthodes en contrôle impulsionnel. Applied Mathematics and Optimization 1 289-312. MR0390886

[20] Bensoussan, A. and Lions, J.-L. (1984). Impulse control and quasivariational inequalities. Gauthier-Villars, Montrouge. Translated from the French by J. M. Cole. MR0756234

[21] Bensoussan, A., Liu, R. H., and Sethi, S. P. (2005). Optimality of an $(s, S)$ policy with compound Poisson and diffusion demands: a quasi-variational inequalities approach. SIAM Journal on Control and Optimization 44 1650-1676. MR2193500

[22] Bertola, G. and Caballero, R. J. (1990). Kinked Adjustment Costs and Aggregate Dynamics. MIT Press, 237-296.

[23] BRAdley, J. R. (2004). A Brownian approximation of a production-inventory system with a manufacturer that subcontracts. Operations Research 52 765-784. MR2091772

[24] BuCKLey, I. R. C. and Korn, R. (1998). Optimal index tracking under transaction costs and impusle control. International Journal of Theoretical and Applied Finance $1315-330$.

[25] Cadenillas, A., Lakner, P., and Pinedo, M. (2010). Optimal control of a meanreverting inventory. Operations Research 58 1697-1710. MR2752714 
[26] Cadenillas, A. and Zapatero, F. (2000) Classical and impulse stochastic control of the exchange rate using interest rates and reserves. Mathematical Finance 10 141156. MR1802595

[27] Cadenillas, A., Zapatero, F. and Sarkar, S. (2007) Optimal dividend policy with mean-reverting cash reservoir. Mathematical Finance 17 81-109. MR2281793

[28] Chaо, H. (1992). The EOQ model with stochatic demand and discounting. European Journal of Operational Research 59 434-443.

[29] Chiarolla, M. B. and Haussmann, U. G. (2005). Explicit solution of a stochastic, irreversible investment problem and its moving threshold. Mathematics of Operations Research 30 91-108. MR2125139

[30] Constantinides, G. M. (1976). Stochastic cash management with fixed and proportional transaction costs. Management Science 22 1320-1331. MR0441249

[31] Constantinides, G. M. and Richard, S. (1978). Existence of optimal simple policies for discounted-cost inventory and cash management in continous time. Operations Research 26 620-636. MR0496558

[32] DAI, J. G. and Lin, W. (2008). Asymptotic optimality of maximum pressure policies in stochastic processing networks. Annals of Applied Probability 18 2239-2299. MR2473656

[33] DAI, J. G. and YAO, D. (2013). Brownian inventory models with convex holding cost, part 2: discount-optimal controls. Stochastic Systems 3(2) 500-573.

[34] Davis, M. H. A. (1993) Markov Models and Optimization. Chapman and Hall, London. MR1283589

[35] Davis, M. H. A. and Zervos, M. (1994). A problem of singular stochastic control with discretionary stopping. The Annals of Applied Probability 4 226-240. MR1258182

[36] Dixit, A. (1991). A Simplified Treatment of the Theory of Optimal Regulation of Brownian motion. Journal of Economic Dynamics \& Control 15 657-673. MR1130779

[37] Egami, M. (2008). A direct solution method for stochastic impulse control problems of one-dimensional diffusions. SIAM Journal on Control and Optimization $471191-$ 1218. MR2407014

[38] Feinberg, E. A. and Lewis, M. E. (2007). Optimality inequalities for average cost Markov decision processes and the stochastic cash balance problem. Mathematics of Operations Research 32 769-783. MR2363196

[39] Feng, H. and Muthuraman, K. (2010). A computational method for stochastic impulse control problems. Mathematics of Operations Research 35 830-850. MR2777517

[40] Harrison, J. M. (1985). Brownian Motion and Stochastic Flow Systems. Wiley, New York. MR0798279

[41] Harrison, J. M. (1998). Heavy traffic analysis of a system with parallel servers: asymptotic analysis of discrete-review policies. Annals of Applied Probability 8 822848. MR1627791

[42] Harrison, J. M. and Lopez, M. J. (1999). Heavy traffic resource pooling in parallelserver systems. Queueing Systems 33 339-368. MR1742575

[43] Harrison, J. M., Sellke, T. M., and Taksar, M. I. (1983). Impulse control of Brownian motion. Mathematics of Operations Research 8 454-466. MR0716124

[44] Harrison, J. M. and TAksar, M. I. (1983). Instantaneous control of Brownian motion. Mathematics of Operations Research 8 439-453. MR0716123 
[45] Harrison, J. M. and TAYlor, A. J. (1978). Optimal control of Brownian storage system. Stochastic Processes and Their Applications 6 179-194. MR0469463

[46] Huh, W. T., Janakiraman, G., and Nagarajan, M. (2011). Average cost singlestage inventory models: An analysis using a vanishing discount approach. Operations Research 59 143-155. MR2814225

[47] IRLE, A. and SASs, J. (2006). Optimal portfolio policies under fixed and proportional transaction costs. Advance in Applied Probability 38 916-942. MR2285688

[48] Jeanblanc-Picque, M. (1993). Impulse control method and exchange rate. Mathematical Finance 3 161-177.

[49] Jeanblanc-Picque, M. and Shiryaev, A. N. (1995) Optimization of the flow of dividends. Russian Mathematical Survey 50 257-277. MR1339263

[50] KorN, R. (1997). Optimal impulse control when control actions have random consequences Mathematics of Operations Research 22 639-667. MR1467390

[51] Korn, R. (1998). Portfolio optimization with strictly positive transaction costs and impulse controls. Finance and Stochastics 2 85-114.

[52] Korn, R. (1999). Some applications of impulse control in mathematical finance. Mathematical Methods of Operations Research 50 493-528. MR1731297

[53] Krichagina, E. V., Lou, S. X. C., and Taksar, M. I. (1994). Double band policy for stochastic manufacturing systems in heavy traffic. Mathematics of Operations Research 19 560-596. MR1288887

[54] Krichagina, E. V., Rubio, R., Taksar, M. I., and Wein, L. W. (1998). A dynamic stochastic stock-cutting problem. Operations Research 46 690-701.

[55] Kumar, S. and Muthuraman, K. (2004). A numerical method for solving singular stochastic control problems. Operations Research 52 563-582. MR2075794

[56] LAKner, P and Reed, J. (2012). Optimal cash management using impulse control. Working paper.

[57] Lon, P. C. and Zervos, M. (2011). A model for optimally advertising and launching a product. Mathematics of Operations Research 36 363-376. MR2828764

[58] Lumley, R. R. and Zervos, M. (2001). A model for investments in the natural resource industry with switching costs. Mathematics of Operations Research $\mathbf{2 6} 637$ 653. MR1870738

[59] Mandelbaum, A. and Stolyar, A. L. (2004). Scheduling flexible servers with convex delay costs: heavy traffic optimality of the generalized $c \mu$-rule. Operations Research 52 836-855. MR2104141

[60] Merhi, A. and Zervos, M. (2007). A model for reversible investment capacity expansion SIAM Journal on Financial Mathematics 46 839-876. MR2338430

[61] Morimoto, H. (2010). A singular control problem with discretionary stopping for geometric Brownian motions. SIAM Journal on Control and Optimization 48 37813804. MR2606836

[62] Mundaca, G. and Oksendal, B. (1998). Optimal stochastic intervention control with application to the exchange rate. Journal of Mathematical Economics 29 225243. MR1614233

[63] Oksendal, B. and Sulem, A. (2002). Optimal consumption and portfolio with both fixed and proportional transaction costs. SIAM Journal on Financial Mathematics 40 1765-1790. MR1897195

[64] Oksendal, B and Sulem, A. (2005) Applied Stochastic Control of Jump Diffusions. Springer-Verlag, Berlin. MR2109687 
[65] Ormeci, M., Dai, J. G., and Vande Vate, J. (2008). Impulse control of Brownian motion: the constrained average cost case. Operations Research 56 618-629. MR2436857

[66] OU, J. and Wein, L. M. (1995). Dynamic scheduling of a production/inventory system with by-products and random yield. Management Science 41 1000-1017.

[67] Paulsen, J. (2007). Optimal dividend payments until ruin of diffusion processes when payments are subject to both fixed and proportional costs. Advance in Applied Probability 39 669-689. MR2357376

[68] Plehn-Dujowich, J. M. (2005). The optimality of a control band policy. Review of Economic Dyanmics 8 877-901.

[69] Pliska, S. and Suzuki, K. (2004). Optimal tracking for asset allocation with fixed and proportional transaction costs. Quantitative Finance 4 233-243. MR2055959

[70] Protter, P. E. (2005). Stochastic Integration and Differential Equations. Stochastic Modelling and Applied Probability, Vol. 21. Springer-Verlag, Berlin. Second edition. Version 2.1, Corrected third printing. MR2273672

[71] Richard, S. F. (1977). Optimal impulse control of a diffusion process with both fixed and proportional costs of control. SIAM Journal on Control and Optimization 15 79-91. MR0490377

[72] Rubino, M. and AtA, B. (2009). Dynamic control of a make-to-order, parallel-server system with cancellations. Operations Research 57 94-108. MR2555589

[73] ScH ̈̈L, M. (1993). Average optimality in dynamic programming with general state space. Mathematics of Operations Research 18 163-172. MR1250112

[74] Stokey, N. L. (2009). The Economics of Inaction. Princeton University Press, Princetion and Oxford.

[75] Sulem, A. (1986). A solvable one-dimensional model of a diffusion inventory system. Mathematics of Operations Research 11 125-133. MR0830113

[76] TAKsar, M. I. (1985). Average optimal singular control and a related stopping problem. Mathematics of Operations Research 10 63-81. MR0787007

[77] Tamura, T. (2008). Maximization of the long-term growth rate for a portfolio with fixed and proportional transaction costs. Advance in Applied Probability 40 673-695. MR2454028

[78] Tsiddon, D. (1993). The (Mis)Behaviour of the Aggregate Price-Level. Review of Economic Studies 60 889-902.

[79] Veatch, M. H. and Wein, L. M. (1996) Scheduling a make-to-stock queue: index policies and hedging points. Operations Research $44634-647$.

[80] Ward, A. R. and Kumar, S. (2008). Asymptotically optimal admission control of a queue with impatient customers. Mathematics of Operations Research 33 167-202. MR2393546

[81] WeIn, L. W. (1992). Dynamic scheduling of a multiclass make-to-stock queue. Operations Research 40 724-735. MR1179800

[82] YAO, D. (2010). Inventory control problem with Brownian motion demand. Ph.D. thesis, Academy of Mathematics and Systems Science, Chinese Academy of Sciences.

School of Operations Research AND INFORMATION ENGINEERING CORnell University

136 ROY ROAD

IthaCA, New York, 14853, U.S.A.

E-MAIL: jim.dai@cornell.edu
Institute of Applied Mathematics ACAdemy of Mathematics and Systems Science Chinese Academy of Sciences No. 55, Zhongguancun East Road Haidian District, Beijing, 100190, China

E-MAIL: dachengyao@amss.ac.cn 